\title{
Direct Trajectory Optimization and Costate Estimation of General Optimal Control Problems Using a Radau Pseudospectral Method
}

\author{
Divya Garg* \\ Michael A. Patterson ${ }^{\dagger}$ \\ Christopher L. Darby ${ }^{\ddagger}$ \\ Camila Francolin ${ }^{\S}$ \\ Department of Mechanical and Aerospace Engineering \\ University of Florida \\ Gainesville, FL 32611 \\ Geoffrey T. Huntington \\ Blue Origin, LLC. \\ Kent, WA 98032 \\ William W. Hager" \\ Anil V. Rao** \\ University of Florida \\ Gainesville, FL 32611
}

\begin{abstract}
A method is presented for direct trajectory optimization and costate estimation using global collocation at Legendre-Gauss-Radau (LGR) points. The method is formulated first by casting the dynamics in integral form and computing the integral from the initial point to the interior LGR points and the terminal point. The resulting integration matrix is nonsingular and thus can be inverted so as to express the dynamics in inverse integral form. Then, by appropriate choice of the approximation for the state, a pseudospectral (i.e., differential) form that is equivalent to the inverse integral form is derived. As a result, the method presented in this paper can be thought of as either a global implicit integration method or a pseudospectral method. Moreover, the formulation derived in this paper enables solving general finite-horizon problems using global collocation at the LGR points. A key feature of the method is that it provides an accurate way to map the KKT multipliers of the nonlinear programming problem (NLP) to the costates of the optimal control problem. Finally,
\end{abstract}

${ }^{*}$ M.S. Student, Dept. of Mechanical and Aerospace Engineering. E-mail: divyagarg2002@ufl.edu

${ }^{\dagger}$ Ph.D. Student, Dept. of Mechanical and Aerospace Engineering. E-mail: mpatterson@ufl.edu

${ }^{\ddagger}$ Ph.D. Student, Dept. of Mechanical and Aerospace Engineering. E-mail: cdarby@ufl.edu

${ }^{\S}$ M.S. Student, Dept. of Mechanical and Aerospace Engineering. E-mail: francoli@ufl.edu

"GN\& C Engineer. E-mail: ghuntington@blue.aero

"Professor, Dept. of Mathematics, E-mail: hager@math.ufl.edu.

**Assistant Professor, Dept. of Mechanical and Aerospace Engineering. E-mail: anilvrao@ufl.edu. Corresponding Author.

1 of 29

American Institute of Aeronautics and Astronautics 
it is shown that a previously developed Radau collocation method, which is restricted to infinite-horizon problems, is subsumed by the method presented in this paper. The results of this paper show that the use of LGR collocation as described in this paper leads to the ability to determine accurate primal and dual solutions to general finite-horizon optimal control problems.

\section{Introduction}

Over the last decade, pseudospectral methods have risen to prominence in the numerical solution of optimal control problems. ${ }^{1-18}$ Pseudospectral methods are a class of direct collocation where the optimal control problem is transcribed to a nonlinear programming problem (NLP) by parameterizing the state and control using global polynomials and collocating the differential-algebraic equations using nodes obtained from a Gaussian quadrature. It is noted that some researchers prefer the term orthogonal collocation, ${ }^{19-21}$ but the terms pseudospectral and orthogonal collocation are the same.

The three most commonly used set of collocation points are Legendre-Gauss (LG), LegendreGauss-Radau (LGR), and Legendre-Gauss-Lobatto (LGL) points. These three sets of points are obtained from the roots of a Legendre polynomial and/or linear combinations of a Legendre polynomial and its derivatives. All three sets of points are defined on the domain $[-1,1]$, but differ significantly in that the LG points include neither of the endpoints, the LGR points include one of the endpoints, and the LGL points include both of the endpoints. In addition, the LGR points are asymmetric relative to the origin and are not unique in that they can be defined using either the initial point or the terminal point. In recent years, the two most well documented pseudospectral methods are the Legendre pseudospectral method ${ }^{1,3-5,10,11,13,14}$ (LPM) and the Gauss pseudospectral method. ${ }^{15-17,22,23}$ With regard to collocation at LGR points, a local collocation method has been developed in Ref. 18 while an LGR method for solving infinite-horizon problems has been developed in Ref. 12 While some work has been done on the topic of collocation at LGR points, LGR collocation still remains the least studied of the pseudospectral methods. In particular, one question that remains about LGR collocation is whether or not it can be used as a global collocation method for solving general finite-horizon problems. A second, somewhat related, question is whether accurate costates can be determined from the KKT multipliers of the NLP that arises from a finite-horizon formulation of LGR collocation.

The purpose of this paper is to describe a method for direct trajectory optimization and costate estimation for general finite-horizon optimal control problems using global collocation at LGR points. In the method presented here, the dynamics are first cast in integral form and the integration points are chosen to be the interior LGR points plus the terminal point. Using the fact that the resulting integration matrix is nonsingular, the problem can be written in inverse integral form. It is then shown that the inverse integral form is equivalent to a pseudospectral form where the state approximation is made using the LGR points plus the terminal point. Thus, the Radau method derived in this paper can be treated equivalently as either a global implicit integration method or a pseudospectral method and can be used to solve problem with general two-point boundary conditions. Furthermore, the method derived here leads to the ability to accurately estimate costates at both the LGR points and the missing endpoint. Finally, the formulation considered in this paper is compared to a previously developed method for solving infinite-horizon optimal control problems using LGR collocation ${ }^{12}$ where it is found that the current formulation subsumes the formulation of Ref. 12. The method presented in this paper is found to be a viable approach for determining accurate primal and dual solutions to general finite-horizon optimal control problems. 


\section{LG, LGR, and LGL Collocation Points}

The LG, LGR, and LGL collocation points lie on the open interval $\tau \in(-1,1)$, the half open interval $\tau \in[-1,1)$ or $\tau \in(-1,1]$, and the closed interval $\tau \in[-1,1]$, respectively. A depiction of these three sets of collocation points is shown in Fig. 1 where it is seen that the LG points contain neither -1 or 1 , the LGR points contain only one of the points -1 or 1 (in this case, the point -1 ), and the LGL points contain both -1 and 1. Denoting $K$ as the number of collocation points and $P_{K}(\tau)$ as the $k^{t h}$-degree Legendre polynomial, the LG points are the roots of $P_{K}(\tau)$, the LGR points are the roots of $P_{K-1}(\tau)+P_{K}(\tau)$, and the LGL points are the roots of $\dot{P}_{K-1}(\tau)$ together with the points -1 and 1 . The polynomials whose roots are the respective points are summarized as follows:

LG: $\quad$ Roots obtained from $P_{K}(\tau)$

LGR: $\quad$ Roots obtained from $P_{K-1}(\tau)+P_{K}(\tau)$

LGL: $\quad$ Roots obtained from $\dot{P}_{k-1}(\tau)$ together with the points -1 and 1

It is seen from Fig. 1 that the LG and LGL points are symmetric about the origin whereas the LGR points are asymmetric. In addition, the LGR points are not unique in that two sets of points exist (one including the point -1 and the other including the point 1). The LGR points that include the terminal endpoint are often called the flipped LGR points. In this paper, however, we use the standard set of LGR points as defined above and consistent with the usage given in Ref. 13.

\section{Continuous Bolza Problem}

Without loss of generality, consider the following optimal control problem in Bolza form. Determine the state, $\mathbf{x}(\tau) \in \mathbb{R}^{n}$, control, $\mathbf{u}(\tau) \in \mathbb{R}^{m}$, initial time, $t_{0}$, and final time, $t_{f}$, that minimize the cost functional

$$
J=\Phi\left(\mathbf{x}\left(\tau_{1}\right), t_{0}, \mathbf{x}\left(\tau_{N}\right), t_{f}\right)+\frac{t_{f}-t_{0}}{2} \int_{-1}^{1} g\left(\mathbf{x}(\tau), \mathbf{u}(\tau), \tau ; t_{0}, t_{f}\right) d \tau
$$

subject to the constraints

$$
\begin{gathered}
\frac{d \mathbf{x}}{d \tau}=\frac{t_{f}-t_{0}}{2} \mathbf{f}\left(\mathbf{x}(\tau), \mathbf{u}(\tau), \tau ; t_{0}, t_{f}\right) \in \mathbb{R}^{n} \\
\phi\left(\mathbf{x}\left(\tau_{1}\right), t_{0}, \mathbf{x}\left(\tau_{N}\right), t_{f}\right)=\mathbf{0} \in \mathbb{R}^{q} \\
\mathbf{C}\left(\mathbf{x}(\tau), \mathbf{u}(\tau), \tau ; t_{0}, t_{f}\right) \leq \mathbf{0} \in \mathbb{R}^{c}
\end{gathered}
$$

The optimal control problem of Eqs. (1)-(4) will be referred to as the continuous Bolza problem. It is noted that the optimal control problem of Eqs. (1)-(4) can be transformed from the time interval $\tau \in[-1,1]$ to the time interval $t \in\left[t_{0}, t_{f}\right]$ via the affine transformation

$$
t=\frac{t_{f}-t_{0}}{2} \tau+\frac{t_{f}+t_{0}}{2}
$$

\section{A. Radau Pseudospectral Discretized Form of Continuous Bolza Problem}

The direct approach for solving the continuous Bolza optimal control problem is given as follows. First, the state is approximated as

$$
\sum_{i=1}^{N} \mathbf{X}_{i} L_{i}(\tau)
$$




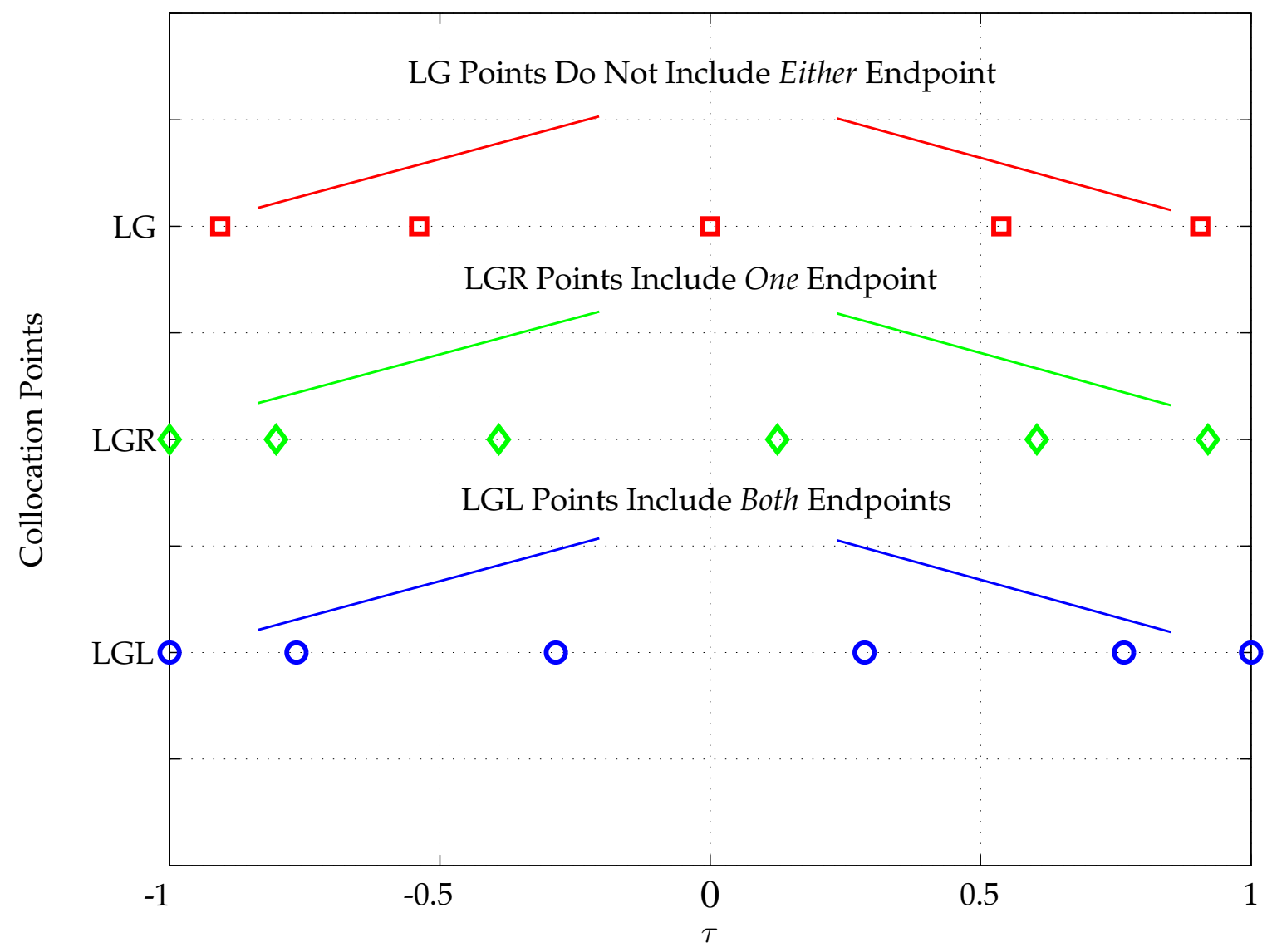

Figure 1: Schematic Showing the Differences Between LGL, LGR, and LG Collocation Points. 
where

$$
L_{i}(\tau)=\prod_{\substack{j=1 \\ j \neq i}}^{N} \frac{\tau-\tau_{j}}{\tau_{i}-\tau_{j}}, \quad(i=1, \ldots, N)
$$

The time derivative of the state approximation of Eq. (6) is then given as

$$
\sum_{i=1}^{N} \mathbf{X}_{i} \dot{L}_{i}(\tau)
$$

The dynamic constraint is then collocated at the $N-1$ LGR points as

$$
\sum_{i=1}^{N} D_{k i} \mathbf{X}_{i}-\frac{t_{f}-t_{0}}{2} \mathbf{f}\left(\mathbf{X}_{k}, \mathbf{U}_{k}, \tau_{k} ; t_{0}, t_{f}\right)=\mathbf{0}, \quad(k=1, \ldots, N-1)
$$

where $D_{k i}=\dot{L}_{k}\left(\tau_{i}\right),(k=1, \ldots, N-1),(i=1, \ldots, N)$ is the $(N-1) \times N$ Radau pseudospectral differentiation matrix, $\mathbf{X}_{k} \equiv \mathbf{X}\left(\tau_{k}\right)$, and $\mathbf{U}_{k} \equiv \mathbf{U}\left(\tau_{k}\right)$. Note that the dynamic constraint is collocated only at the LGR points whereas the state is approximated at the $N-1$ LGR points $p l u s$ the terminal point, $\tau_{N}=1$. Next, the continuous-time cost functional of Eq. (1) is approximated using a Gauss-Radau quadrature as

$$
J=\Phi\left(\mathbf{X}_{1}, t_{0}, \mathbf{X}_{N}, t_{f}\right)+\frac{t_{f}-t_{0}}{2} \sum_{k=1}^{N-1} w_{k} g\left(\mathbf{X}_{k}, \mathbf{U}_{k}, \tau_{k} ; t_{0}, t_{f}\right)
$$

where $w_{k},(k=1, \ldots, N-1)$ are the LGR weights. Furthermore, similar to the way that the endpoint cost is applied at the boundary points, the continuous-time boundary conditions of Eq. (3) are also approximated at the boundary points as

$$
\phi\left(\mathbf{X}_{1}, t_{0}, \mathbf{X}_{N}, t_{f}\right)=\mathbf{0}
$$

Lastly, the path constraint of Eq. (4) is evaluated at the LGR points as

$$
\mathbf{C}\left(\mathbf{X}_{k}, \mathbf{U}_{k}, \tau_{k} ; t_{0}, t_{f}\right) \leq \mathbf{0}, \quad(k=1, \ldots, N-1)
$$

The cost function of Eq. (9) along with the algebraic constraints of Eqs. (8),(10) and (11) define an NLP whose solution is an approximate solution to the continuous Bolza problem of Section III.

\section{B. KKT Conditions of the NLP}

The KKT conditions or the first-order optimality conditions of the NLP are obtained as follows. First, the augmented cost function is formed using Lagrange multipliers $\tilde{\Lambda}_{k} \in \mathbb{R}^{n}, \tilde{\boldsymbol{\mu}}_{k} \in \mathbb{R}^{c}(k=$ $1, \ldots, N-1)$ and $\tilde{\boldsymbol{\nu}} \in \mathbb{R}^{q}$ as

$$
\begin{aligned}
J_{a} & =\Phi\left(\mathbf{X}_{1}, t_{0}, \mathbf{X}_{N}, t_{f}\right)+\frac{t_{f}-t_{0}}{2} \sum_{k=1}^{N-1} w_{k} g\left(\mathbf{X}_{k}, \mathbf{U}_{k}, \tau_{k} ; t_{0}, t_{f}\right) \\
& -\tilde{\boldsymbol{\nu}}^{T} \boldsymbol{\phi}\left(\mathbf{X}_{1}, t_{0}, \mathbf{X}_{N}, t_{f}\right)-\sum_{k=1}^{N-1} \tilde{\boldsymbol{\mu}}_{k}^{T} \mathbf{C}\left(\mathbf{X}_{k}, \mathbf{U}_{k}, \tau_{k} ; t_{0}, t_{f}\right) \\
& -\sum_{k=1}^{N-1} \tilde{\boldsymbol{\Lambda}}_{k}^{T}\left(\sum_{i=1}^{N} D_{k i} \mathbf{X}_{i}-\frac{t_{f}-t_{0}}{2} \mathbf{f}\left(\mathbf{X}_{k}, \mathbf{U}_{k}, \tau_{k} ; t_{0}, t_{f}\right)\right)
\end{aligned}
$$


The KKT conditions of the NLP are then obtained by setting equal to zero the derivatives of the Lagrangian taken with respect to : $\mathbf{X}_{1}, \mathbf{X}_{N}, \mathbf{X}_{k}, \mathbf{U}_{k}, \tilde{\boldsymbol{\Lambda}}_{k}, \tilde{\boldsymbol{\nu}}, \tilde{\boldsymbol{\mu}}_{k}, t_{0}$, and $t_{f}$, resulting in the following conditions:

$$
\begin{aligned}
& \phi\left(\mathbf{X}_{1}, t_{0}, \mathbf{X}_{N}, t_{f}\right)=\mathbf{0} \\
& \frac{\partial g_{k}}{\partial \mathbf{U}_{k}}+\left(\frac{\tilde{\boldsymbol{\Lambda}}_{k}}{w_{k}}\right)^{T} \frac{\partial \mathbf{f}_{k}}{\partial \mathbf{U}_{k}}-\frac{2}{t_{f}-t_{0}}\left(\frac{\tilde{\boldsymbol{\mu}}_{k}}{w_{k}}\right)^{T} \frac{\partial \mathbf{C}_{k}}{\partial \mathbf{U}_{k}}=\mathbf{0}, \quad(k=1, \ldots, N-1) \\
& \sum_{i=1}^{N} D_{k i} \mathbf{X}_{i}-\left(\frac{t_{f}-t_{0}}{2}\right) \mathbf{f}\left(\mathbf{X}_{k}, \mathbf{U}_{k}, \tau_{k} ; t_{0}, t_{f}\right)=\mathbf{0}, \quad(k=1, \ldots, N-1) \\
& \frac{\partial \Phi}{\partial t_{0}}-\tilde{\boldsymbol{\nu}}^{T} \frac{\partial \phi}{\partial t_{0}}=-\frac{t_{f}-t_{0}}{2} \sum_{k=1}^{N-1} w_{k}\left[\frac{\partial g_{k}}{\partial t_{0}}+\left(\frac{\tilde{\boldsymbol{\Lambda}}_{k}}{w_{k}}\right)^{T} \frac{\partial \mathbf{f}_{k}}{\partial t_{0}}-\frac{2}{t_{f}-t_{0}}\left(\frac{\tilde{\boldsymbol{\mu}}_{k}}{w_{k}}\right)^{T} \frac{\partial \mathbf{C}_{k}}{\partial t_{0}}\right] \\
& +\frac{1}{2} \sum_{i=1}^{N-1} w_{k}\left[g_{k}+\left(\frac{\tilde{\boldsymbol{\Lambda}}_{k}}{w_{k}}\right)^{T} \mathbf{f}_{k}-\frac{2}{t_{f}-t_{0}}\left(\frac{\tilde{\boldsymbol{\mu}}_{k}}{w_{k}}\right)^{T} \mathbf{C}_{k}\right] \\
& -\frac{\partial \Phi}{\partial t_{f}}+\tilde{\boldsymbol{\nu}}^{T} \frac{\partial \boldsymbol{\phi}}{\partial t_{f}}=\frac{t_{f}-t_{0}}{2} \sum_{k=1}^{N-1} w_{k}\left[\frac{\partial g_{k}}{\partial t_{f}}+\left(\frac{\tilde{\boldsymbol{\Lambda}}_{k}}{w_{k}}\right)^{T} \frac{\partial \mathbf{f}_{k}}{\partial t_{f}}-\frac{2}{t_{f}-t_{0}}\left(\frac{\tilde{\boldsymbol{\mu}}_{k}}{w_{k}}\right)^{T} \frac{\partial \mathbf{C}_{k}}{\partial t_{f}}\right] \\
& +\frac{1}{2} \sum_{i=1}^{N-1} w_{k}\left[g_{k}+\left(\frac{\tilde{\boldsymbol{\Lambda}}_{k}}{w_{k}}\right)^{T} \mathbf{f}_{k}-\frac{2}{t_{f}-t_{0}}\left(\frac{\tilde{\boldsymbol{\mu}}_{k}}{w_{k}}\right)^{T} \mathbf{C}_{k}\right] \\
& \mathbf{C}\left(\mathbf{X}_{k}, \mathbf{U}_{k}, \tau_{k} ; t_{0}, t_{f}\right) \leq \mathbf{0}, \quad(k=1, \ldots, N-1) \\
& \tilde{\boldsymbol{\mu}}_{j k}=0 \text { when } C_{j k}<0, \quad(j=1, . ., c ; k=1, . ., N-1) \\
& \tilde{\boldsymbol{\mu}}_{j k} \leq 0 \text { when } C_{j k}=0, \quad(j=1, . ., c ; k=1, . ., N-1) \\
& \frac{t_{f}-t_{0}}{2}\left[\frac{\partial g_{1}}{\partial \mathbf{X}_{1}}+\left(\frac{\tilde{\boldsymbol{\Lambda}}_{1}}{w_{1}}\right)^{T} \frac{\partial \mathbf{f}_{1}}{\partial \mathbf{X}_{1}}-\frac{2}{t_{f}-t_{0}}\left(\frac{\tilde{\boldsymbol{\mu}}_{1}}{w_{1}}\right)^{T} \frac{\partial \mathbf{C}_{1}}{\partial \mathbf{U}_{1}}\right]^{T}+\sum_{i=1}^{N-1} \frac{\tilde{\boldsymbol{\Lambda}}_{i}}{w_{i}} D_{1 i}^{\dagger}=-\frac{1}{w_{1}}\left[\frac{\tilde{\boldsymbol{\Lambda}}_{1}}{w_{1}}+\left(\frac{\partial \Phi}{\partial \mathbf{X}_{1}}-\tilde{\boldsymbol{\nu}}^{T} \frac{\partial \phi}{\partial \mathbf{X}_{1}}\right)^{T}\right] \\
& \frac{t_{f}-t_{0}}{2}\left[\frac{\partial g_{k}}{\partial \mathbf{X}_{k}}+\left(\frac{\tilde{\boldsymbol{\Lambda}}_{k}}{w_{k}}\right)^{T} \frac{\partial \mathbf{f}_{k}}{\partial \mathbf{X}_{k}}-\frac{2}{t_{f}-t_{0}}\left(\frac{\tilde{\boldsymbol{\mu}}_{k}}{w_{k}}\right)^{T} \frac{\partial \mathbf{C}_{k}}{\partial \mathbf{X}_{k}}\right]^{T}+\sum_{i=1}^{N-1}\left(\frac{\tilde{\boldsymbol{\Lambda}}_{i}}{w_{i}}\right) D_{k i}^{\dagger}=\mathbf{0}, \quad(k=2, \ldots, N-1) \\
& \left(\frac{\partial \Phi}{\partial \mathbf{X}_{N}}-\tilde{\boldsymbol{\nu}}^{T} \frac{\partial \phi}{\partial \mathbf{X}_{N}}\right)^{T}-\sum_{i=1}^{N-1} \tilde{\boldsymbol{\Lambda}}_{i} D_{i N}=\mathbf{0}
\end{aligned}
$$

Now, defining the quantity $\tilde{\Lambda}_{N}$ as

$$
\tilde{\boldsymbol{\Lambda}}_{N}=\sum_{i=1}^{N-1} \tilde{\boldsymbol{\Lambda}}_{i} D_{i N}
$$

Eq. (23) can be re-written as

$$
\left(\frac{\partial \Phi}{\partial \mathbf{X}_{N}}-\tilde{\boldsymbol{\nu}}^{T} \frac{\partial \phi}{\partial \mathbf{X}_{N}}\right)^{T}-\tilde{\boldsymbol{\Lambda}}_{N}=\mathbf{0}
$$


Using the relationships given by Eq. (45)-(47), Eq. (24) can be expressed in an alternate manner as follows:

$$
\begin{aligned}
\tilde{\boldsymbol{\Lambda}}_{N} & =\sum_{i=1}^{N-1} \tilde{\boldsymbol{\Lambda}}_{i} D_{i N} \\
& =-\sum_{i=1}^{N-1} \tilde{\boldsymbol{\Lambda}}_{i} \sum_{k=1}^{N-1} D_{i k}=-\sum_{i=1}^{N-1} \sum_{k=1}^{N-1} \tilde{\boldsymbol{\Lambda}}_{i} D_{i k} \\
& =-\sum_{i=1}^{N-1}\left(\tilde{\boldsymbol{\Lambda}}_{i} D_{i 1}+\sum_{k=2}^{N-1} \tilde{\boldsymbol{\Lambda}}_{i} D_{i k}\right)=-\sum_{i=1}^{N-1} \tilde{\boldsymbol{\Lambda}}_{i} D_{i 1}-\sum_{i=1}^{N-1} \sum_{k=2}^{N-1} \tilde{\boldsymbol{\Lambda}}_{i} D_{i k} \\
& =-\tilde{\boldsymbol{\Lambda}}_{1} D_{11}-\sum_{i=2}^{N-1} \tilde{\boldsymbol{\Lambda}}_{i} D_{i 1}-\sum_{k=2}^{N-1} \sum_{i=1}^{N-1} \tilde{\boldsymbol{\Lambda}}_{i} D_{i k} \\
& =-\tilde{\boldsymbol{\Lambda}}_{1}\left(-D_{11}^{\dagger}-\frac{1}{w_{1}}\right)+\sum_{i=2}^{N-1} \tilde{\boldsymbol{\Lambda}}_{i} \frac{w_{1}}{w_{i}} D_{1 i}^{\dagger}+\sum_{k=2}^{N-1} w_{k} \sum_{i=1}^{N-1} \frac{\tilde{\boldsymbol{\Lambda}}_{i}}{w_{i}} D_{k i}^{\dagger} \\
& =\frac{\tilde{\boldsymbol{\Lambda}}_{1}}{w_{1}}+\tilde{\boldsymbol{\Lambda}}_{1} D_{11}^{\dagger}+\sum_{i=2}^{N-1} \tilde{\boldsymbol{\Lambda}}_{i} \frac{w_{1}}{w_{i}} D_{1 i}^{\dagger}+\sum_{k=2}^{N-1} w_{k} \sum_{i=1}^{N-1} \frac{\tilde{\boldsymbol{\Lambda}}_{i}}{w_{i}} D_{k i}^{\dagger} \\
& =\frac{\tilde{\boldsymbol{\Lambda}}_{1}}{w_{1}}+w_{1} \sum_{i=1}^{N-1} \frac{\tilde{\boldsymbol{\Lambda}}_{i}}{w_{i}} D_{1 i}^{\dagger}+\sum_{k=2}^{N-1} w_{k} \sum_{i=1}^{N-1} \frac{\tilde{\boldsymbol{\Lambda}}_{i}}{w_{i}} D_{k i}^{\dagger}=\frac{\tilde{\boldsymbol{\Lambda}}_{1}}{w_{1}}+\sum_{k=1}^{N-1} w_{k} \sum_{i=1}^{N-1} \frac{\tilde{\boldsymbol{\Lambda}}_{i}}{w_{i}} D_{k i}^{\dagger} \\
& \frac{\tilde{\boldsymbol{\Lambda}}_{1}}{w_{1}}+\frac{t_{f}-t_{0}}{2} \sum_{k=1}^{N-1} w_{k}\left[-\frac{\partial g_{k}}{\partial \mathbf{X}_{k}}-\left(\frac{\tilde{\boldsymbol{\Lambda}}_{k}}{w_{k}}\right)^{T} \frac{\partial \mathbf{f}_{k}}{\partial \mathbf{X}_{k}}+\frac{2}{t_{f}-t_{0}}\left(\frac{\tilde{\boldsymbol{\mu}}_{k}}{w_{k}}\right)^{T} \frac{\partial \mathbf{C}_{k}}{\partial \mathbf{X}_{k}}\right]^{T}
\end{aligned}
$$

Equation (26) will be used in Section C to derive a mapping between the KKT multipliers and the costates.

\section{Radau Pseudospectral Method for Discretizing Continuous Bolza Problem}

We now derive a pseudospectral method for solving general optimal control problems using global collocation at Legendre-Gauss-Radau points. The derivation of described below will be divided into four parts: (i) the first-order optimality condtions of the continuous Bolza problem; (ii) the Radau pseudospectral discretization of the continuous-time first-order optimality conditions of the continuous Bolza problem; (iii) the Radau pseudospectral discretization of the continuoustime optimal control problem, resulting in a discrete NLP; (iv) the KKT conditions of the NLP; (v) a costate estimation obtained from the results of (iii) and (iv).

\section{A. First-Order Necessary Conditions of the Continuous Bolza Problem}

The indirect approach for solving the continuous Bolza optimal control problem of Eqs. (1)-(4) given in Section III is to apply the calculus of variation and Pontryagin's maximum principle to obtain first-order necessary conditions for optimality. First, the augmented Hamiltonian is defined as

$$
H\left(\mathbf{x}, \boldsymbol{\lambda}, \mathbf{u}, \boldsymbol{\mu}, \tau ; t_{0}, t_{f}\right)=g\left(\mathbf{x}, \mathbf{u}, \tau ; t_{0}, t_{f}\right)+\boldsymbol{\lambda}^{T}(\tau) \mathbf{f}\left(\mathbf{x}, \mathbf{u}, \tau ; t_{0}, t_{f}\right)-\boldsymbol{\mu}^{T}(\tau) \mathbf{C}\left(\mathbf{x}, \mathbf{u}, \tau ; t_{0}, t_{f}\right)
$$


where $\boldsymbol{\lambda}(\tau) \in \mathbb{R}^{n}$ is the costate and $\boldsymbol{\mu}(\tau) \in \mathbb{R}^{c}$ is the Lagrange multiplier associated with the path constraint. The continuous-time first-order optimality conditions are then given as $^{24}$

$$
\begin{gathered}
\frac{\partial H}{\partial \mathbf{u}}=\frac{\partial g}{\partial \mathbf{u}}+\boldsymbol{\lambda}^{T} \frac{\partial \mathbf{f}}{\partial \mathbf{u}}-\boldsymbol{\mu}^{T} \frac{\partial \mathbf{C}}{\partial \mathbf{u}}=\mathbf{0} \\
\frac{d \mathbf{x}}{d \tau}=\frac{t_{f}-t_{0}}{2} \frac{\partial H^{T}}{\partial \boldsymbol{\lambda}}=\frac{t_{f}-t_{0}}{2} \mathbf{f}\left(\mathbf{x}, \mathbf{u}, \tau ; t_{0}, t_{f}\right) \\
\frac{d \boldsymbol{\lambda}}{d \tau}=-\frac{t_{f}-t_{0}}{2}\left(\frac{\partial H}{\partial \mathbf{x}}\right)^{T}=-\frac{t_{f}-t_{0}}{2}\left(\frac{\partial g}{\partial \mathbf{x}}+\boldsymbol{\lambda}^{T} \frac{\partial \mathbf{f}}{\partial \mathbf{x}}-\boldsymbol{\mu}^{T} \frac{\partial \mathbf{C}}{\partial \mathbf{x}}\right)^{T} \\
\boldsymbol{\phi}\left(\mathbf{x}\left(t_{0}\right), t_{0}, \mathbf{x}\left(t_{f}\right), t_{f}\right)=\mathbf{0} \\
\boldsymbol{\lambda}^{T}\left(\tau_{1}\right)=-\frac{\partial \Phi}{\partial \mathbf{x}\left(\tau_{0}\right)}+\boldsymbol{\nu}^{T} \frac{\partial \phi}{\partial \mathbf{x}\left(\tau_{0}\right)}, \quad \boldsymbol{\lambda}^{T}\left(\tau_{N}\right)=\frac{\partial \Phi}{\partial \mathbf{x}\left(\tau_{N}\right)}-\boldsymbol{\nu}^{T} \frac{\partial \phi}{\partial \mathbf{x}\left(\tau_{N}\right)} \\
H\left(\tau_{1}\right)=\frac{\partial \Phi}{\partial t_{0}}-\boldsymbol{\nu}^{T} \frac{\partial \phi}{\partial t_{0}}, \quad H\left(\tau_{N}\right)=-\frac{\partial \Phi}{\partial t_{f}}+\boldsymbol{\nu}^{T} \frac{\partial \phi}{\partial t_{f}}
\end{gathered}
$$

where $\nu \in \mathbb{R}^{q}$ is the Lagrange multiplier associated with the boundary condition $\phi$. It can be shown that the augmented Hamiltonian at the initial and final times can be written, respectively, as

$$
\begin{aligned}
& H\left(\tau_{1}\right)=-\frac{t_{f}-t_{0}}{2} \int_{-1}^{1} \frac{\partial H}{\partial t_{0}} d \tau+\frac{1}{2} \int_{-1}^{1} H d \tau \\
& H\left(\tau_{N}\right)=\frac{t_{f}-t_{0}}{2} \int_{-1}^{1} \frac{\partial H}{\partial t_{f}} d \tau+\frac{1}{2} \int_{-1}^{1} H d \tau
\end{aligned}
$$

\section{B. Radau Pseudospectral Discretized First-Order Necessary Conditions of Continuous Bolza Problem}

The first-order conditions for optimality are discretized using the Radau pseudospectral method as follows. First, the state is approximated in a manner consistent with Eq. (6) using the $N-1$ LGR points plus the terminal point, $\tau_{N}=1$, as

$$
\mathbf{x}(\tau) \approx \mathbf{X}(\tau)=\sum_{i=1}^{N} \mathbf{X}\left(\tau_{i}\right) \tilde{L}_{i}(\tau)
$$

where the Lagrange polynomials $\tilde{L}_{i}(\tau)(i=1, \ldots, N)$ are defined as

$$
\tilde{L}_{i}(\tau)=\prod_{\substack{j=1 \\ j \neq i}}^{N} \frac{\tau-\tau_{j}}{\tau_{i}-\tau_{j}}
$$

The time derivative of the state approximation given in Eq. (36) is then obtained as

$$
\dot{\mathbf{x}}(\tau) \approx \dot{\mathbf{X}}(\tau)=\sum_{i=1}^{N} \dot{\tilde{L}}_{i}(\tau) \mathbf{X}\left(\tau_{i}\right)
$$

Applying the time derivative of Eq. (38) at the $N-1$ LGR points $\left(\tau_{1}, \ldots, \tau_{N-1}\right)$ gives

$$
\dot{\mathbf{x}}\left(\tau_{k}\right) \approx \dot{\mathbf{X}}\left(\tau_{k}\right)=\sum_{i=1}^{N} \dot{\tilde{L}}_{i}\left(\tau_{k}\right) \mathbf{X}\left(\tau_{i}\right)=\sum_{i=1}^{N} D_{k i} \mathbf{X}\left(\tau_{i}\right), \quad(k=1, \ldots, N-1)
$$


where $D_{k i},(k=1, \ldots, N-1 ; i=1, \ldots, N)$ is the $(N-1) \times N R P M$ differentiation matrix. Next, the costate is approximated as

$$
\boldsymbol{\lambda}(\tau) \approx \boldsymbol{\Lambda}(\tau)=\sum_{i=1}^{N-1} L_{i}^{\dagger}(\tau) \boldsymbol{\Lambda}\left(\tau_{i}\right)
$$

where the Lagrange polynomials $L_{i}^{\dagger}(\tau)(i=1, \ldots, N-1)$ are defined as

$$
L_{i}^{\dagger}(\tau)=\prod_{\substack{j=1 \\ j \neq i}}^{N-1} \frac{\tau-\tau_{j}}{\tau_{i}-\tau_{j}}
$$

It is noted here that the costate approximation differs from the state approximation in that the costate approximation does not include the terminal point $\tau_{N}=1$. As a result, the interpolation points for the costate are only the values of the costate at the LGR points and do not include the value of the costate at the terminal time. The time derivative of the costate is then given as

$$
\dot{\boldsymbol{\lambda}}(\tau) \approx \dot{\boldsymbol{\Lambda}}(\tau)=\sum_{i=1}^{N-1} \dot{L}_{i}^{\dagger}(\tau) \boldsymbol{\Lambda}\left(\tau_{i}\right)
$$

The approximation to the time derivative of the costate is then applied at the $N-1$ LGR collocation points $\left(\tau_{1}, \ldots, \tau_{N-1}\right)$ as

$$
\dot{\boldsymbol{\lambda}}\left(\tau_{k}\right) \approx \dot{\boldsymbol{\Lambda}}\left(\tau_{k}\right)=\sum_{i=1}^{N-1} \dot{L}_{i}^{\dagger}\left(\tau_{k}\right) \boldsymbol{\Lambda}\left(\tau_{i}\right)=\sum_{i=1}^{N-1} D_{k i}^{\dagger} \boldsymbol{\Lambda}\left(\tau_{i}\right), \quad(k=1, \ldots, N-1)
$$

where $D_{k i}^{\dagger},(k=1, \ldots, N-1 ; i=1, \ldots, N-1)$ is the $(N-1) \times(N-1)$ adjoint RPM differentiation matrix. The matrices $D$ and $D^{\dagger}$ are related to one another by the following result:

Theorem 1 The matrices $D_{k i}$ and $D_{i k}^{\dagger}$ defined in Eqs. (39) and (43), respectively, are related to one another as

$$
\begin{gathered}
D_{11}=-D_{11}^{\dagger}-\frac{1}{w_{1}} \\
D_{i k}=-\frac{w_{k}}{w_{i}} D_{k i}^{\dagger}, \quad(i, k=1, . ., N-1 ; i \neq k) \\
D_{i i}=-D_{i i}^{\dagger}, \quad(i=2, \ldots, N-1) \\
D_{i N}=-\sum_{k=1}^{N-1} D_{i k}, \quad(i=1, \ldots, N-1)
\end{gathered}
$$

where $w_{k},(k=1, \ldots, N-1)$ are the LGR weights.

Proof of Theorem 1 Consider the integration by parts formula

$$
\int_{-1}^{1} \dot{p}(\tau) q(\tau) d \tau=[p(\tau) q(\tau)]_{-1}^{1}-\int_{-1}^{1} p(\tau) \dot{q}(\tau) d \tau
$$


Approximating the integrals in Eq. (48) using a Gauss-Radau quadrature, we have

$$
\begin{aligned}
& w_{1} q(-1) \sum_{l=1}^{N} D_{1 l} q\left(\tau_{l}\right)+\sum_{j=2}^{N-1} \sum_{l=1}^{N} D_{j l} p\left(\tau_{l}\right) q\left(\tau_{j}\right) w_{j} \\
& =p(1) q(1)-p(-1) q(-1)-w_{1} p(-1) \sum_{l=1}^{N-1} D_{1 l}^{\dagger} q\left(\tau_{l}\right)-\sum_{j=2}^{N-1} \sum_{l=1}^{N-1} D_{j l}^{\dagger} q\left(\tau_{l}\right) p\left(\tau_{j}\right) w_{j}
\end{aligned}
$$

Equation (49) must hold for all polynomials $p(\tau)$ of degree $N-1$ or less and all polynomials $q(\tau)$ of degrees $N-2$ or less. Consequently, Eq. (49) must hold for for the set of $(N-1)^{\text {th }}$-degree Lagrange polynomials $\tilde{L}_{k}(\tau),(k=1, \ldots, N)$ defined in Eq. (37) and the set of $(N-2)^{t h}$-degree Lagrange polynomials $\tilde{L}_{k}(\tau),(k=1, \ldots, N-1)$ defined in Eq. (41). Substituting these Lagrange polynomials into Eq. (49), we obtain

$$
\begin{aligned}
& w_{1} L_{i}^{\dagger}(-1) \sum_{l=1}^{N} D_{1 l} \tilde{L}_{k}\left(\tau_{l}\right)+\sum_{j=2}^{N-1} \sum_{l=1}^{N} D_{j l} \tilde{L}_{k}\left(\tau_{l}\right) L_{i}^{\dagger}\left(\tau_{j}\right) w_{j} \\
& =\tilde{L}_{k}(1) L_{i}^{\dagger}(1)-\tilde{L}_{k}(-1) L_{i}^{\dagger}(-1)-w_{1} \tilde{L}_{k}(-1) \sum_{l=1}^{N-1} D_{1 l}^{\dagger} L_{i}^{\dagger}\left(\tau_{l}\right)-\sum_{j=2}^{N-1} \sum_{l=1}^{N-1} D_{j l}^{\dagger} \tilde{L}_{k}\left(\tau_{j}\right) L_{i}^{\dagger}\left(\tau_{l}\right) w_{j}
\end{aligned}
$$

For $i=1$ we have

$$
\begin{aligned}
& L_{1}^{\dagger}(-1)=1 \\
& L_{1}^{\dagger}\left(\tau_{j}\right)=0, \quad(j=2, \ldots, N-1)
\end{aligned}
$$

Eq. (50) then reduces to

$$
w_{1} \sum_{l=1}^{N} D_{1 l} \tilde{L}_{k}\left(\tau_{l}\right)=\tilde{L}_{k}(1) L_{i}^{\dagger}(1)-\tilde{L}_{k}(-1)-w_{1} D_{11}^{\dagger} \tilde{L}_{k}(-1)-\sum_{j=2}^{N-1} D_{j 1}^{\dagger} \tilde{L}_{k}\left(\tau_{j}\right) w_{j}
$$

Now for $k=1$ we have

$$
\begin{aligned}
& \tilde{L}_{1}(-1)=1 \\
& \tilde{L}_{1}\left(\tau_{j}\right)=0, \quad(j=2, \ldots, N)
\end{aligned}
$$

Equation (52) then reduces to

$$
w_{1} D_{11}=-1-w_{1} D_{11}^{\dagger}
$$

which implies that

$$
D_{11}=-D_{11}^{\dagger}-\frac{1}{w_{1}}
$$

Next, for $i=1$ and $k=2, \ldots, N-1$ we have from Eq. (52) that

$$
w_{1} D_{1 k} \tilde{L}_{k}\left(\tau_{k}\right)=-w_{k} D_{k 1}^{\dagger} \tilde{L}_{k}\left(\tau_{k}\right)
$$

Then, knowing that $\tilde{L}_{k}\left(\tau_{k}\right)=1,(k=2, \ldots, N-1)$, we obtain

$$
w_{1} D_{1 k}=-w_{k} D_{k 1}^{\dagger}
$$

from which we obtain

$$
D_{1 k}=-\frac{w_{k}}{w_{1}} D_{k 1}^{\dagger}
$$

10 of 29 
Next, substituting $i=2, \ldots, N-1$ ane $k=2, \ldots, N-1$ into Eq. (50) gives

$$
\sum_{j=2}^{N-1} \sum_{l=1}^{N} D_{j l} \tilde{L}_{k}\left(\tau_{l}\right) L_{i}^{\dagger}\left(\tau_{j}\right) w_{j}=-\sum_{j=2}^{N-1} \sum_{l=1}^{N-1} D_{j l}^{\dagger} \tilde{L}_{k}\left(\tau_{j}\right) L_{i}^{\dagger}\left(\tau_{l}\right) w_{j}
$$

Now we know that

$$
\begin{array}{ll}
\tilde{L}_{k}\left(\tau_{l}\right) L_{i}^{\dagger}\left(\tau_{j}\right)=0 \quad & \quad(l \neq k ; j \neq 1) \\
\tilde{L}_{k}\left(\tau_{j}\right) L_{i}^{\dagger}\left(\tau_{l}\right)=0 \quad & (j \neq k ; l \neq i)
\end{array}
$$

Therefore,

$$
D_{i k} w_{i}=-D_{k i}^{\dagger} w_{k}
$$

which implies that

$$
D_{i k}=-\frac{w_{k}}{w_{i}} D_{k i}^{\dagger}
$$

Combining Eqs. (58) and (62) gives

$$
\begin{aligned}
& D_{i k}=-\frac{w_{k}}{w_{i}} D_{k i}^{\dagger}, \quad(i, k=1, \ldots, N-1 ; i \neq k) \\
& D_{i i}=-D_{i i}^{\dagger}, \quad(i=2, \ldots, N-1)
\end{aligned}
$$

Lastly, suppose that $f(\tau)=c$ where $c$ is a constant. Then we have

$$
\dot{f}\left(\tau_{i}\right)=\sum_{k=1}^{N-1} D_{i k} f\left(\tau_{i}\right)+D_{i N} f\left(\tau_{N}\right)=\sum_{k=1}^{N-1} D_{i k} c+D_{i N} c=0
$$

Therefore,

$$
D_{i N}=-\sum_{k=1}^{N-1} D_{i k}
$$

Using the state and costate approximation as given in Eqs. (36) and (40), the first-order necessary conditions of the continuous Bolza problem in Section A are discretized as follows. The continuous-time first-order optimality conditions of Section A are discretized using the variables $\mathbf{X}_{k} \equiv \mathbf{X}\left(\tau_{k}\right) \in \mathbb{R}^{n}$ and $\mathbf{X}_{N} \equiv \mathbf{X}\left(\tau_{N}\right)$ for the state, $\mathbf{U}_{k} \equiv \mathbf{U}\left(\tau_{k}\right) \in \mathbb{R}^{m}$ for the control, $\boldsymbol{\Lambda}_{k} \equiv \boldsymbol{\Lambda}\left(\tau_{k}\right) \in \mathbb{R}^{n}$ and $\boldsymbol{\Lambda}_{N} \equiv \boldsymbol{\Lambda}\left(\tau_{N}\right)$ for the costate and $\boldsymbol{\mu}_{k} \equiv \boldsymbol{\mu}\left(\tau_{k}\right) \in \mathbb{R}^{c}$ for the Lagrange multiplier associated with the path constraints at the LGR points $k=1,2, . ., N-1$. The other unknown variables in the problem are the initial time, $t_{0} \in \mathbb{R}$, the final time, $t_{f} \in \mathbb{R}$ and the Lagrange multiplier $\boldsymbol{\nu} \in \mathbb{R}^{q}$. These variables are used to discretize the continuous necessary conditions of Section A via the Radau pseudospectral discretization. The resulting algebraic equations that approximate the continuous necessary conditions at the LGR points are given as

$$
\begin{gathered}
\sum_{i=1}^{N} D_{k i} \mathbf{X}_{i}-\left(\frac{t_{f}-t_{0}}{2}\right) \mathbf{f}\left(\mathbf{X}_{k}, \mathbf{U}_{k}, \tau_{k} ; t_{0}, t_{f}\right)=\mathbf{0}, \quad(k=1, \ldots, N-1) \\
\sum_{i=1}^{N-1} D_{k i}^{\dagger} \boldsymbol{\Lambda}_{i}=\frac{t_{f}-t_{0}}{2}\left[-\frac{\partial g_{k}}{\partial \mathbf{X}_{k}}-\boldsymbol{\Lambda}_{k}^{T} \frac{\partial \mathbf{f}_{k}}{\partial \mathbf{X}_{k}}+\boldsymbol{\mu}_{k}^{T} \frac{\partial \mathbf{C}_{k}}{\partial \mathbf{X}_{k}}\right]^{T}, \quad(k=1, \ldots, N-1) \\
\frac{\partial g_{k}}{\partial \mathbf{U}_{k}}+\boldsymbol{\Lambda}_{k}^{T} \frac{\partial \mathbf{f}_{k}}{\partial \mathbf{U}_{k}}-\boldsymbol{\mu}_{k}^{T} \frac{\partial \mathbf{C}_{k}}{\partial \mathbf{U}_{k}}=\mathbf{0}, \quad(k=1, \ldots, N-1)
\end{gathered}
$$




$$
\begin{gathered}
\boldsymbol{\phi}\left(\mathbf{X}_{1}, t_{0}, \mathbf{X}_{N}, t_{f}\right)=\mathbf{0} \\
\boldsymbol{\Lambda}_{1}^{T}=-\left(\frac{\partial \Phi}{\partial \mathbf{X}_{1}}-\boldsymbol{\nu}^{T} \frac{\partial \boldsymbol{\phi}}{\partial \mathbf{X}_{1}}\right) \\
\boldsymbol{\Lambda}_{N}^{T}=\frac{\partial \Phi}{\partial \mathbf{X}_{N}}-\boldsymbol{\nu}^{T} \frac{\partial \boldsymbol{\phi}}{\partial \mathbf{X}_{N}} \\
\frac{\partial \Phi}{\partial t_{0}}-\tilde{\boldsymbol{\nu}}^{T} \frac{\partial \phi}{\partial t_{0}}=-\frac{t_{f}-t_{0}}{2} \sum_{k=1}^{N-1} w_{k}\left[\frac{\partial g_{k}}{\partial t_{0}}+\boldsymbol{\Lambda}_{k}^{T} \frac{\partial \mathbf{f}_{k}}{\partial t_{0}}-\boldsymbol{\mu}_{k}^{T} \frac{\partial \mathbf{C}_{k}}{\partial t_{0}}\right] \\
+\frac{1}{2} \sum_{i=1}^{N-1} w_{k}\left[g_{k}+\boldsymbol{\Lambda}_{k}^{T} \mathbf{f}_{k}-\boldsymbol{\mu}_{k}^{T} \mathbf{C}_{k}\right] \\
-\frac{\partial \Phi}{\partial t_{f}}+\tilde{\boldsymbol{\nu}}^{T} \frac{\partial \phi}{\partial t_{f}}=\frac{t_{f}-t_{0}}{2} \sum_{k=1}^{N-1} w_{k}\left[\frac{\partial g_{k}}{\partial t_{0}}+\mathbf{\Lambda}_{k}^{T} \frac{\partial \mathbf{f}_{k}}{\partial t_{0}}-\boldsymbol{\mu}_{k}^{T} \frac{\partial \mathbf{C}_{k}}{\partial t_{0}}\right] \\
+\frac{1}{2} \sum_{i=1}^{N-1} w_{k}\left[g_{k}+\boldsymbol{\Lambda}_{k}^{T} \mathbf{f}_{k}-\boldsymbol{\mu}_{k}^{T} \mathbf{C}_{k}\right] \\
\mathbf{C}\left(\mathbf{X}_{k}, \mathbf{U}_{k}, \tau_{k} ; t_{0}, t_{f}\right) \leq \mathbf{0}, \quad(k=1, \ldots, N-1) \\
\tilde{\boldsymbol{\mu}}_{j k}=0 \text { when } C_{j k}<0, \quad(j=1, . ., c ; k=1, . ., N-1) \\
\tilde{\boldsymbol{\mu}}_{j k} \leq 0 \text { when } C_{j k}=0, \quad(j=1, . ., c ; k=1, . ., N-1)
\end{gathered}
$$

Finally, Gauss-Radau quadrature is used to write an equation relating the initial and final costate as

$$
\boldsymbol{\Lambda}_{N}=\boldsymbol{\Lambda}_{1}+\left(\frac{t_{f}-t_{0}}{2}\right) \sum_{k=1}^{N-1} w_{k}\left[-\frac{\partial g_{k}}{\partial \mathbf{X}_{k}}-\boldsymbol{\Lambda}_{k}^{T} \frac{\partial \mathbf{f}_{k}}{\partial \mathbf{X}_{k}}+\boldsymbol{\mu}_{k}^{T} \frac{\partial \mathbf{C}_{k}}{\partial \mathbf{X}_{k}}\right]^{T}
$$

The total number of variables in this system of equations are $2 N n+(m+c)(N-1)+q+2$ whereas the total number of equations in this system are $(2 N+1) n+(m+c)(N-1)+q+2$. Clearly, we have an over-determined system of equations here with more number of equations than the number of variables to be solved for.

\section{Costate and Lagrange Multipliers Estimate}

Using the results of Sections B and B, a costate estimate at the LGR points and the boundary points and a Lagrange multipliers estimate associated with the boundary condition and path constraints for the continuous Bolza problem is now obtained. A costate estimate at the final time and the LGR points can be found from the KKT multipliers $\tilde{\boldsymbol{\Lambda}}_{k}, \tilde{\boldsymbol{\Lambda}}_{N}, \tilde{\boldsymbol{\mu}}_{k}$ and $\tilde{\nu}$,

$$
\begin{gathered}
\boldsymbol{\Lambda}_{k}=\frac{\tilde{\boldsymbol{\Lambda}}_{k}}{w_{k}}, \quad(k=1, \ldots, N-1) \\
\boldsymbol{\Lambda}_{N}=\tilde{\boldsymbol{\Lambda}}_{N} \\
\boldsymbol{\nu}=\tilde{\boldsymbol{\nu}} \\
\boldsymbol{\mu}_{k}=\frac{2}{t_{f}-t_{0}} \frac{\tilde{\boldsymbol{\mu}}_{k}}{w_{k}}, \quad(k=1, \ldots, N-1)
\end{gathered}
$$


It is seen that Eqs. (13)-(26) are identical to Eqs. (66)-(77), with the one exception of Eq. (21) where the transversality condition at the initial time is mixed with the costate dynamics collocated at the initial time. Comparing the KKT conditions with the Radau Pseudospectral discretized necessary conditions of Section B, it is seen that, with the exception of the KKT multiplier at the initial time, all of the remaining KKT conditions are equivalent to the discretized form of the continuous firstorder necessary conditions of the continuous Bolza problem when using the Radau pseudospectral discretization. The left hand side of Eq. (21) is the costate dynamic constraint collocated at the initial point while the right hand side of Eq. (21) is the transversality condition obtained from first-order necessary conditions for optimality of the continuous Bolza problem. It is seen that the initial costate does not exactly match with that obtained from the initial transversality condition, but the final costate is the same as obtained from the transversality condition.

\section{Radau Pseudospectral Discretization of Infinite-Horizon Problems}

Consider the following optimal control problem. Minimize the infinite-horizon cost functional

$$
J=\int_{0}^{\infty} g(\mathbf{x}(t), \mathbf{u}(t), t) d t
$$

subject to the dynamic constraint

$$
\dot{\mathbf{x}}=\mathbf{f}(\mathbf{x}(t), \mathbf{u}(t), t)
$$

with the initial condition

$$
\mathbf{x}(0)=\mathbf{x}_{0} .
$$

Consider further the following transformation of time found in Ref. 12:

$$
t=\frac{1+\tau}{1-\tau}
$$

This transformation maps the interval $t \in[0, \infty)$ to the closed interval $\tau \in[-1,1]$. Using (85), the infinite-horizon optimal control problem (82)-(84) can be written in terms of $\tau$ as follows. Minimize the cost functional

$$
J=\int_{-1}^{1} \frac{2}{(1-\tau)^{2}} g(\mathbf{x}(\tau), \mathbf{u}(\tau), \tau) d \tau
$$

subject to the dynamic constraint

$$
\frac{d \mathbf{x}}{d \tau}=\frac{2}{(1-\tau)^{2}} \mathbf{f}(\mathbf{x}(\tau), \mathbf{u}(\tau), \tau)
$$

with the initial condition

$$
\mathbf{x}(-1)=\mathbf{x}_{0}
$$

The transformed infinite-horizon optimal control problem (86)-(88) can be solved using the following modification of the Radau pseudospectral discretization. Minimize the cost function

$$
J=\sum_{k=1}^{N} \frac{2 w_{k}}{\left(1-\tau_{k}\right)^{2}} g\left(\mathbf{X}_{k}, \mathbf{U}_{k}, \tau_{k}\right)
$$


subject to the constraints

$$
\begin{gathered}
\sum_{i=1}^{N} D_{k i} \mathbf{X}_{i}=\frac{2}{\left(1-\tau_{i}\right)^{2}} \mathbf{f}\left(\mathbf{X}_{i}, \mathbf{U}_{i}, \tau_{i} ; t_{0}, t_{f}\right), \quad(k=1, \ldots, N) \\
\mathbf{X}_{1}=\mathbf{x}_{0}
\end{gathered}
$$

It is noted in the NLP of $(89)-(\mathrm{V})$ that the state is approximated at the LGR points plus the terminal point (at $\tau=1$ ). Hence we obtain an approximation of the state at the horizon $t=\infty$. Moreover, the NLP avoids the singularity at $\tau=+1$ in the factor $2 /(1-\tau)^{2}$ because $\tau_{k}=+1$ is not a quadrature point. As is discussed in Section VII below, the solution obtained using the Radau pseudospectral method of this paper differs fundamentally from the infinite-horizon method given in Ref. 12 because in the method of Ref. 12 the state is obtained only at the LGR points whereas in the method presented here the state is obtained at the LGR points and the terminal point $\tau=+1$.

\section{Examples}

In this section we consider two examples using the aforementioned Radau pseudospectral method. The first example is a nonlinear one-dimensional finite-horizon optimal control problem taken from Ref. 15 while the second example is an infinite-horizon linear quadratic problem taken from Ref. 12. It is noted that these two examples utilize the finite-horizon and infinite-horizon forms of the Radau pseudospectral method, respectively.

\section{Example 1: Nonlinear One-Dimensional Finite-Horizon Problem}

Consider the following optimal control problem. Minimize the cost functional

$$
J=\frac{1}{2} \int_{0}^{t_{f}}\left(y+u^{2}\right) d t
$$

subject to the dynamic constraint

$$
\dot{y}=2 y+2 u \sqrt{y}
$$

and the boundary conditions

$$
\begin{aligned}
& y(0)=2, \\
& y\left(t_{f}\right)=1, \\
& t_{f}=5 .
\end{aligned}
$$

It is noted that the exact solution to the optimal control problem of (92)-(94) is given as

$$
\begin{aligned}
& y^{*}(t)=x^{2}(t) \\
& \lambda_{y}^{*}(t)=\frac{\lambda_{x}}{2 \sqrt{y}}
\end{aligned}
$$

where $x(t)$ and $\lambda_{x}(t)$ are given as

$$
\left[\begin{array}{c}
x(t) \\
\lambda_{x}(t)
\end{array}\right]=\exp (\mathbf{A} t)\left[\begin{array}{c}
x_{0} \\
\lambda_{x 0}
\end{array}\right]
$$


where

$$
\begin{aligned}
\mathbf{A} & =\left[\begin{array}{cc}
1 & -1 \\
-1 & -1
\end{array}\right] \\
x_{0} & =\sqrt{2} \\
x_{f} & =1 \\
\lambda_{x 0} & =\frac{x_{f}-B_{11} x_{0}}{B_{12}}
\end{aligned}
$$

and

$$
\mathbf{B}=\left[\begin{array}{ll}
B_{11} & B_{12} \\
B_{21} & B_{22}
\end{array}\right]=\exp \left(\mathbf{A} t_{f}\right)
$$

Example 1 was solved using the Radau pseudospectral method (RPM) with the software OptimalPrime $^{26}$ and the NLP solver SNOPT ${ }^{27}$ for $N=4$ to $N=99$ LGR points. The SNOPT optimality

and feasibility tolerances were $10^{-10}$. A typical solution for $N=39$ LGR points (i.e., $N+1=40$ discretization points) is shown in Fig. 2 alongside the exact solution. Suppose now that we define the following maximum absolute errors between the RPM solution and the exact solution:

$$
\begin{aligned}
e_{y} & =\max _{k \in[1, \ldots, N+1]} \log _{10}\left|y\left(\tau_{k}\right)-y^{*}\left(\tau_{k}\right)\right| \\
e_{\lambda_{y}} & =\max _{k \in[1, \ldots, N+1]} \log _{10}\left|\lambda_{y}\left(\tau_{k}\right)-\lambda_{y}^{*}\left(\tau_{k}\right)\right| \\
e_{u} & =\max _{k \in[1, \ldots, N]} \log _{10}\left|u\left(\tau_{k}\right)-u^{*}\left(\tau_{k}\right)\right|
\end{aligned}
$$

Figs. 3-4 show $e_{y}, e_{u}$, and $e_{\lambda_{y}}$ as a function of $N+1$. It is seen that $e_{y}, e_{u}$, and $e_{\lambda_{y}}$ decrease in a linear manner from $N=4$ to 49 . Moreover, for $N \geq 50$ all three errors remain essentially constant, $e_{y}$ and $e_{u}$ being constant at approximately $10^{-10}$ and $e_{u}$ being constant at approximately $10^{-9}$. The rate of decrease of $e$ for the lower number of nodes is most revealing because it shows that $e$ decreases linearly, demonstrating a spectral convergence rate.

\section{Example 2: Infinite-Horizon LQR Problem}

Consider the following optimal control problem taken from Ref. 12. Denoting $\mathbf{x}(t)=\left[x_{1}(t) x_{2}(t)\right]^{T} \in$ $\mathbb{R}^{2}$ as the state and $u(t) \in \mathbb{R}$ as the control, minimize the cost functional

$$
J=\frac{1}{2} \int_{0}^{\infty}\left(\mathbf{x}^{T} \mathbf{Q} \mathbf{x}+\mathbf{u}^{T} \mathbf{R u}\right) d t
$$

subject to the dynamic constraint

$$
\dot{\mathbf{x}}=\mathbf{A x}+\mathbf{B u}
$$

and the initial condition

$$
\mathbf{x}(0)=\left[\begin{array}{c}
-4 \\
4
\end{array}\right]
$$

The matrices $\mathbf{A}, \mathbf{B}, \mathbf{Q}$, and $\mathbf{R}$ for this problem are given as

$$
\mathbf{A}=\left[\begin{array}{rr}
0 & 1 \\
2 & -1
\end{array}\right], \mathbf{B}=\left[\begin{array}{l}
0 \\
1
\end{array}\right], \quad \mathbf{Q}=\left[\begin{array}{ll}
2 & 0 \\
0 & 1
\end{array}\right], \quad \mathbf{R}=\frac{1}{2}
$$




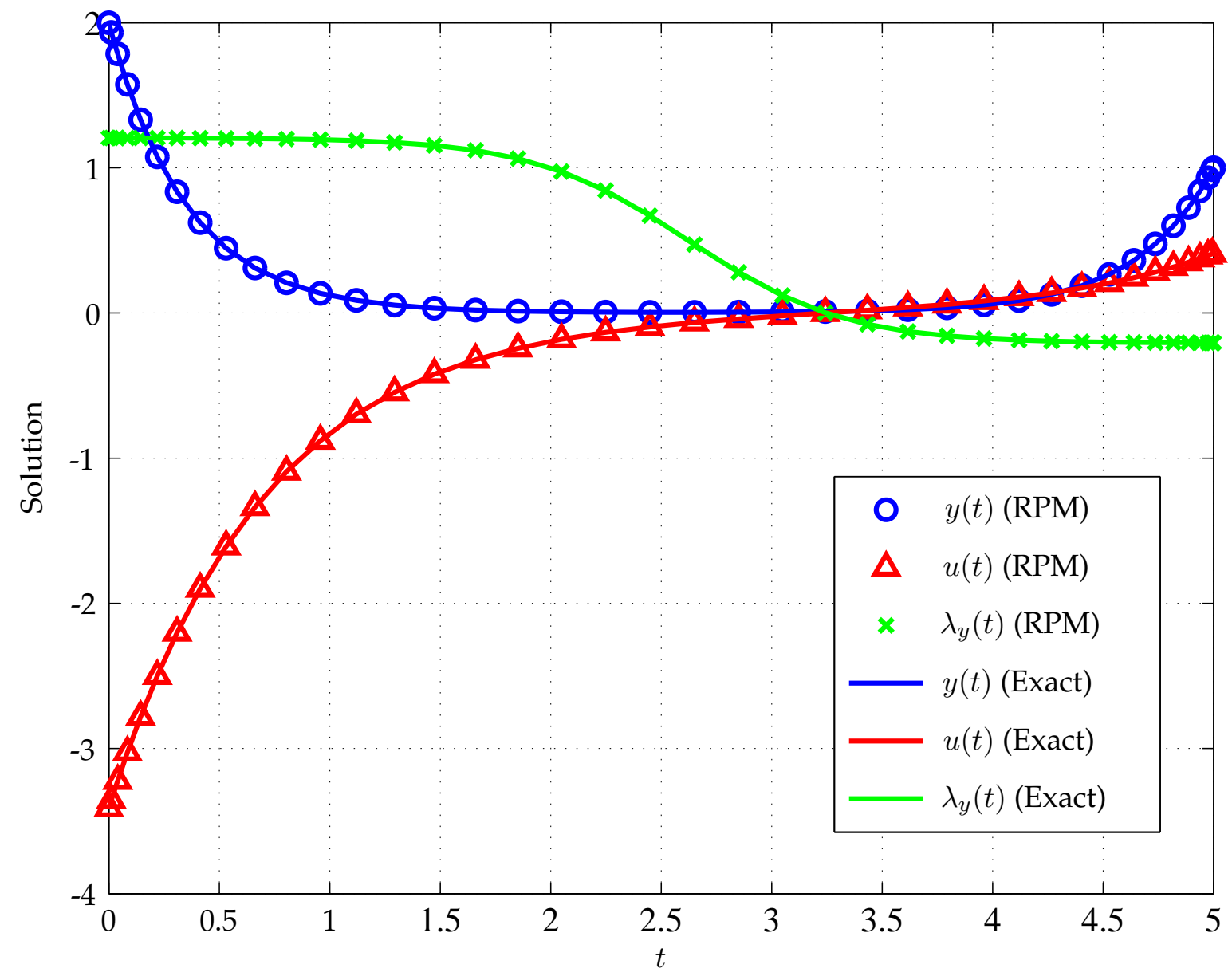

Figure 2: Solution to Example 1 Using 39 LGR Points Alongside Exact Solution. 


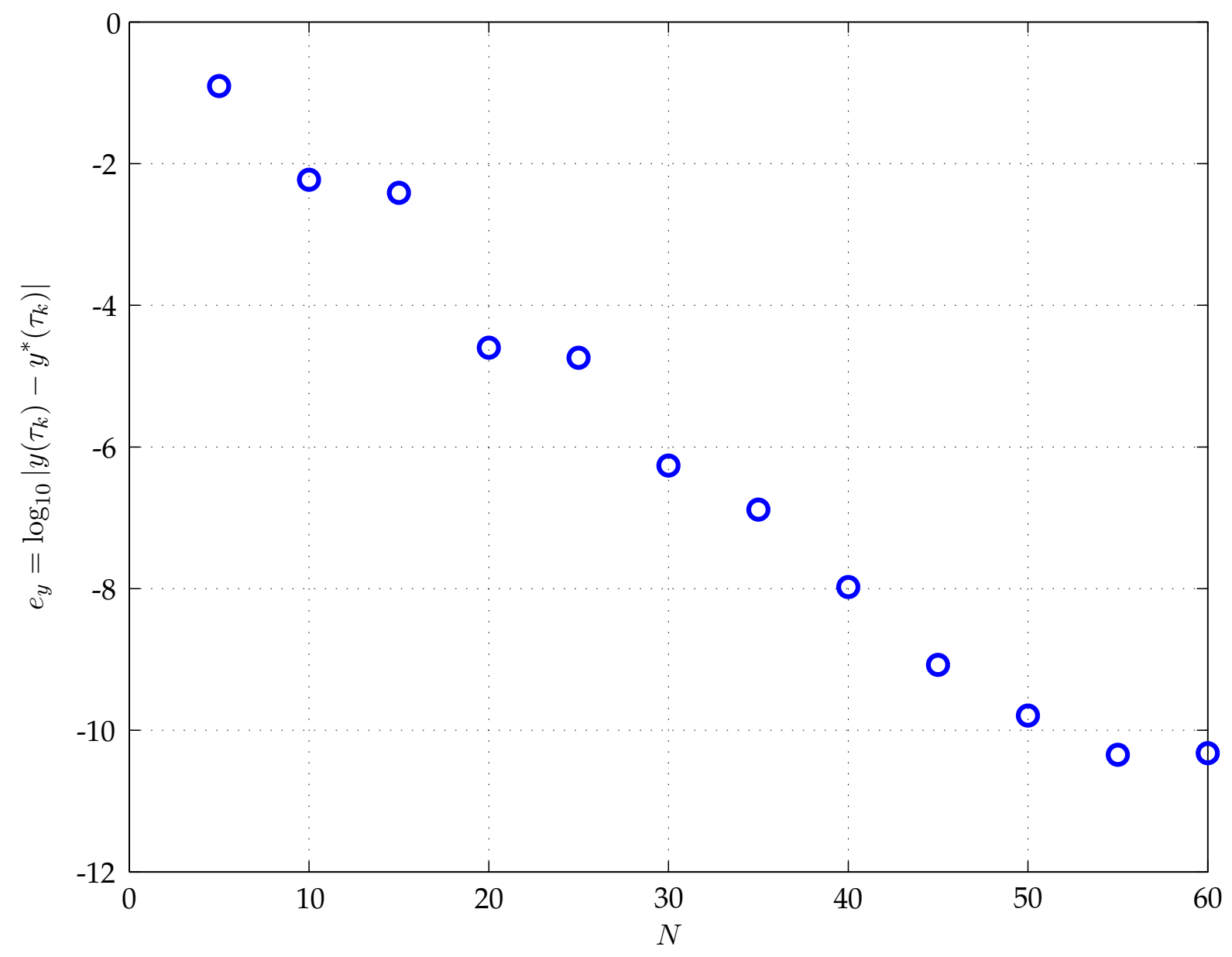

Figure 3: Error in Radau Pseudospectral State for Example 1. 


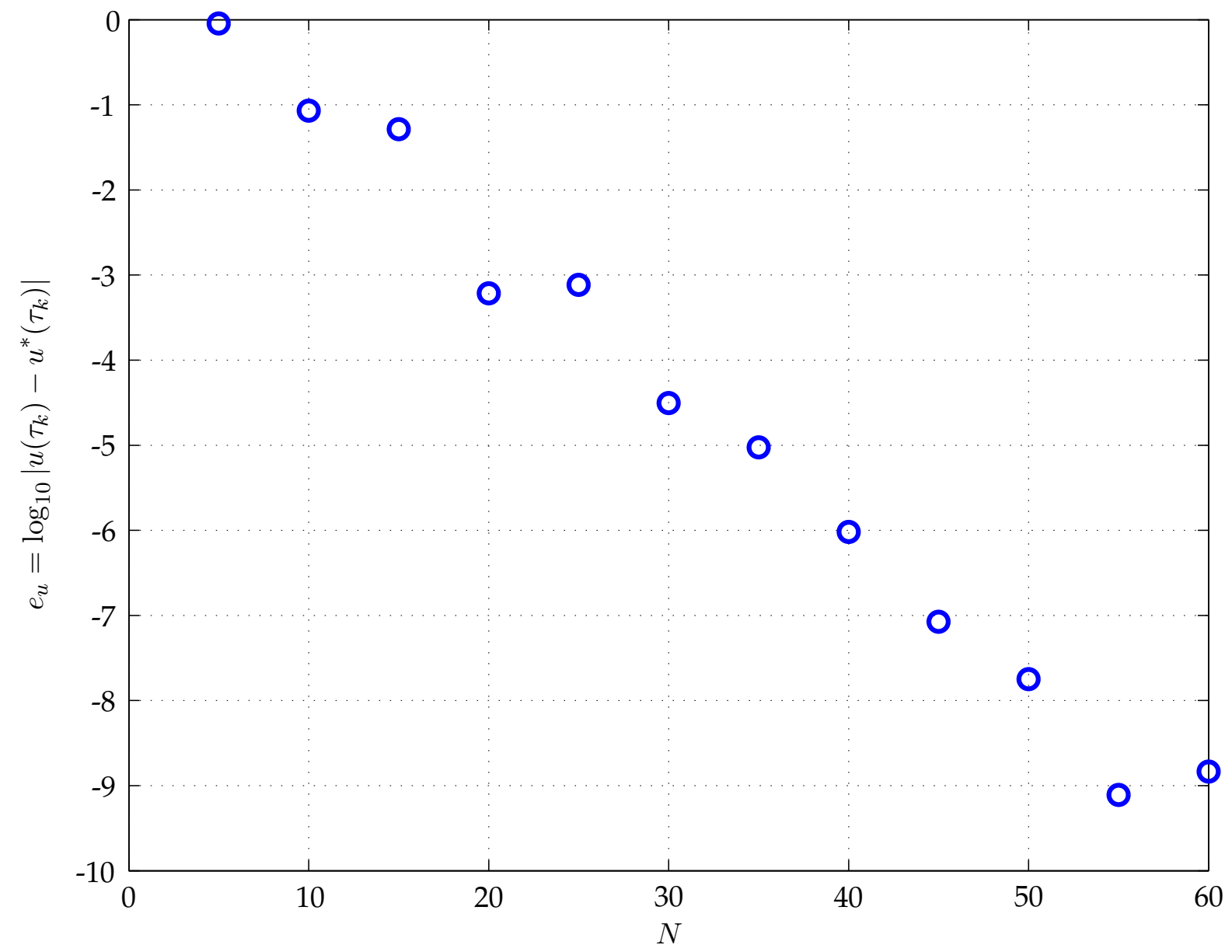

Figure 4: Error in Radau Pseudospectral Control for Example 1. 


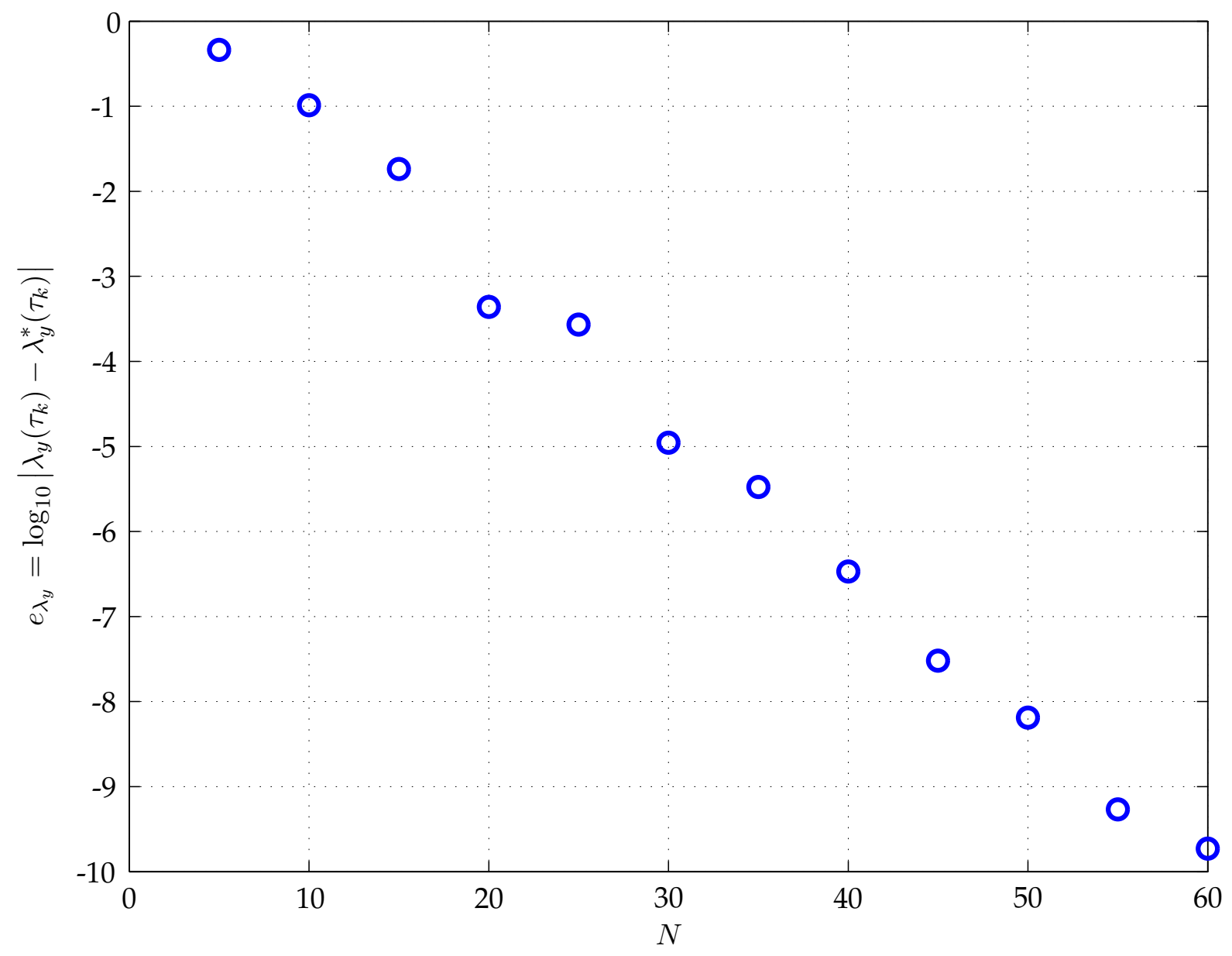

Figure 5: Error in Radau Pseudospectral Costate for Example 1. 
The exact solution to this problem is

$$
\begin{aligned}
\mathbf{x}(t) & =\exp ([\mathbf{A}-\mathbf{B K}] t) \mathbf{x}(0) \\
u(t) & =-\mathbf{K} \mathbf{x}(t) \\
\boldsymbol{\lambda}(t) & =\mathbf{S x}(t)
\end{aligned}
$$

where $\mathbf{K}$ is the optimal feedback gain and $\mathbf{S}$ is the solution to the algebraic Riccati equation. In this case $\mathbf{K}$ and $\mathbf{S}$ are given, respectively, as

$$
\begin{aligned}
& \mathbf{K}=\left[\begin{array}{ll}
4.828427124746193 & 2.557647291327851
\end{array}\right] \\
& \mathbf{S}=\left[\begin{array}{ll}
6.031273049535752 & 2.414213562373097 \\
2.414213562373097 & 1.278823645663925
\end{array}\right]
\end{aligned}
$$

The optimal control problem of Eqs. (100)-(102) was solved using the infinite-horizon version of the Radau pseudospectral method (as given in Section V) using the software OptimalPrime ${ }^{26}$ and the NLP solver SNOPT ${ }^{27}$ with default optimality and feasibility tolerances of $10^{-6}$ and $2 \times 10^{-6}$, respectively, for $N=5$ to $N=35$ by steps of 5 . The infinite-horizon RPM solution for $N=35$ is shown in Figs 6-7 as a function of $\tau$ alongside the exact solution. It is seen that the RPM solution and the exact solution are indistinguishable for all three quantities (state, control, and costate). In particular, it is seen that the infinite horizon version of the RPM solves the problem at all of the LGR points plus the point $\tau=+1$ (i.e., $t=\infty$ ), thus computing the solution on the infinite horizon. Suppose now that we define the following maximum absolute errors between the RPM solution and the exact solution:

$$
\begin{aligned}
e_{\mathbf{x}} & =\max _{k \in[1, \ldots, N+1]} \log _{10}\left|\mathbf{x}\left(\tau_{k}\right)-\mathbf{x}^{*}\left(\tau_{k}\right)\right| \\
e_{u} & =\max _{k \in[1, \ldots, N]} \log _{10}\left|u\left(\tau_{k}\right)-u^{*}\left(\tau_{k}\right)\right| \\
e_{\boldsymbol{\lambda}} & =\max _{k \in[1, \ldots, N+1]} \log _{10}\left|\boldsymbol{\lambda}\left(\tau_{k}\right)-\boldsymbol{\lambda}^{*}\left(\tau_{k}\right)\right|
\end{aligned}
$$

The values of $e_{\mathbf{x}}, e_{\boldsymbol{\lambda}_{y}}$, and $e_{\mathbf{u}}$ are shown in Figs. 9-11. It is seen that all errors decrease linearly until approximately $N=35$, again demonstrating a spectral convergence rate.

\section{Comparison with Previous Work on LGR Collocation}

It is noted that two earlier LGR collocation methods have been derived. The first of these methods is given in Ref. 18 and focuses on local collocation using LGR points. The second method is that given in Ref. 12 and describes a global method for solving infinite-horizon problems. In this section we comment briefly on how the method derived in this paper relates to each of these previously derived methods.

\section{A. Comparison with Local LGR Collocation Method of Ref. 18}

The method derived in this paper shares similarities with the method of Ref. 18 in that the approximation of the state uses the same basis of Lagrange polynomials. It is noted, however, that the method of Ref. 18 uses local collocation, favoring a small number of collocation points and many subintervals (called finite elements in Ref. 18) whereas the current method uses a large number of collocation points and a single interval (i.e., global collocation). As a result, the method of Ref. 18 


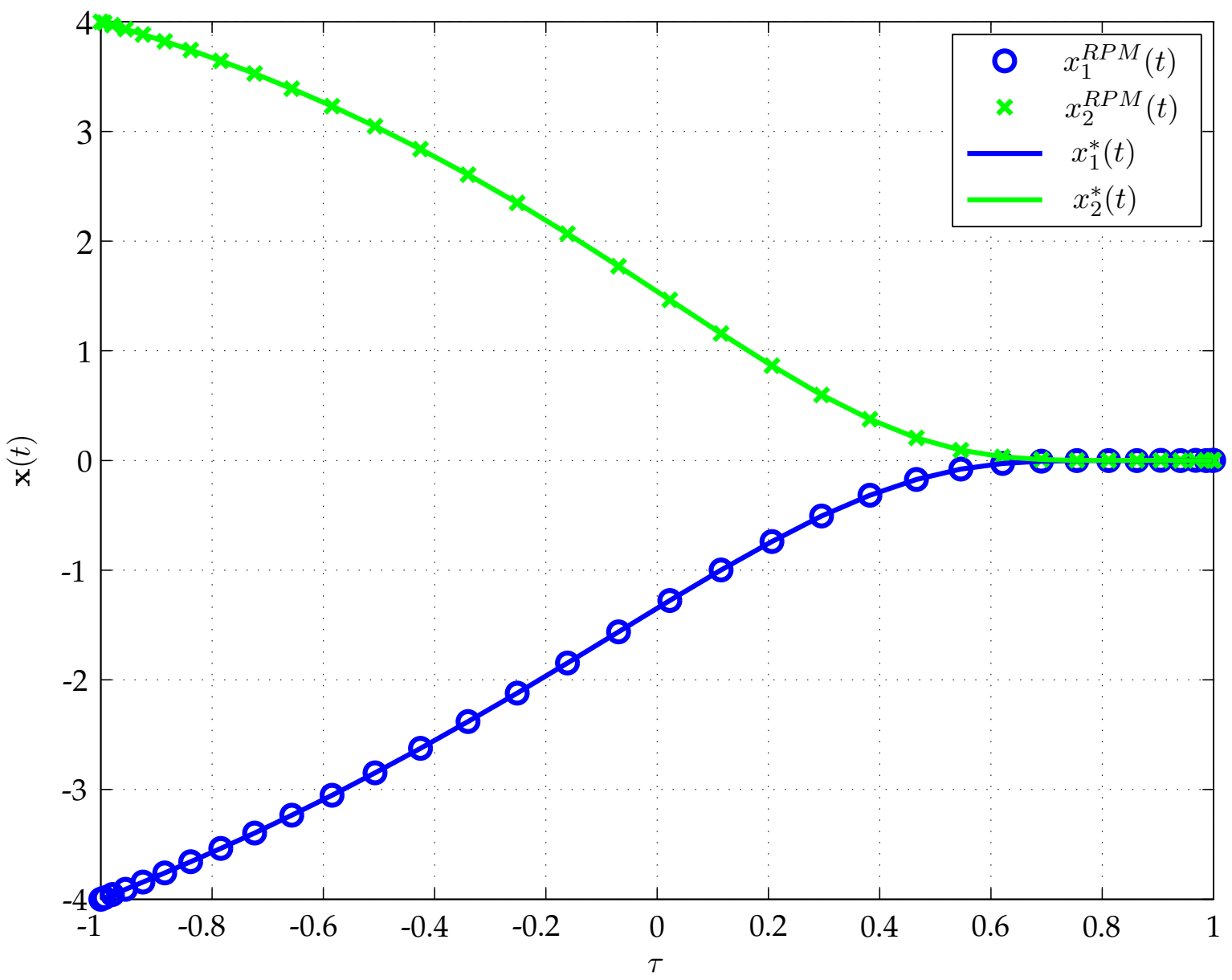

Figure 6: Infinite-Horizon Radau Pseudospectral State Solution for Example 2 Using $N=35$ as a Function of $\tau$ Alongside Exact Solution. 


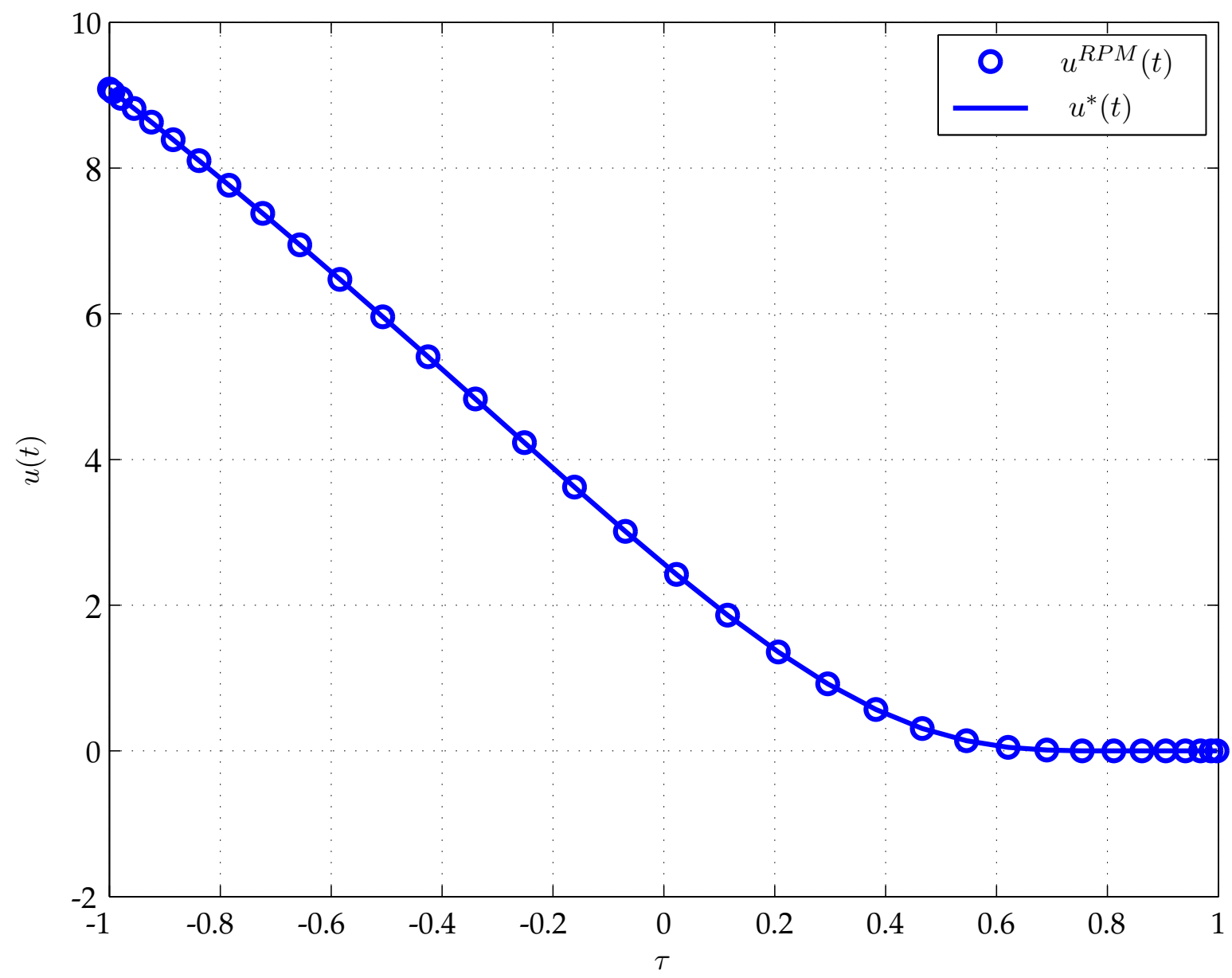

Figure 7: Infinite-Horizon Radau Pseudospectral Control Solution for Example 2 Using $N=35$ as a Function of $\tau$ Alongside Exact Solution. 


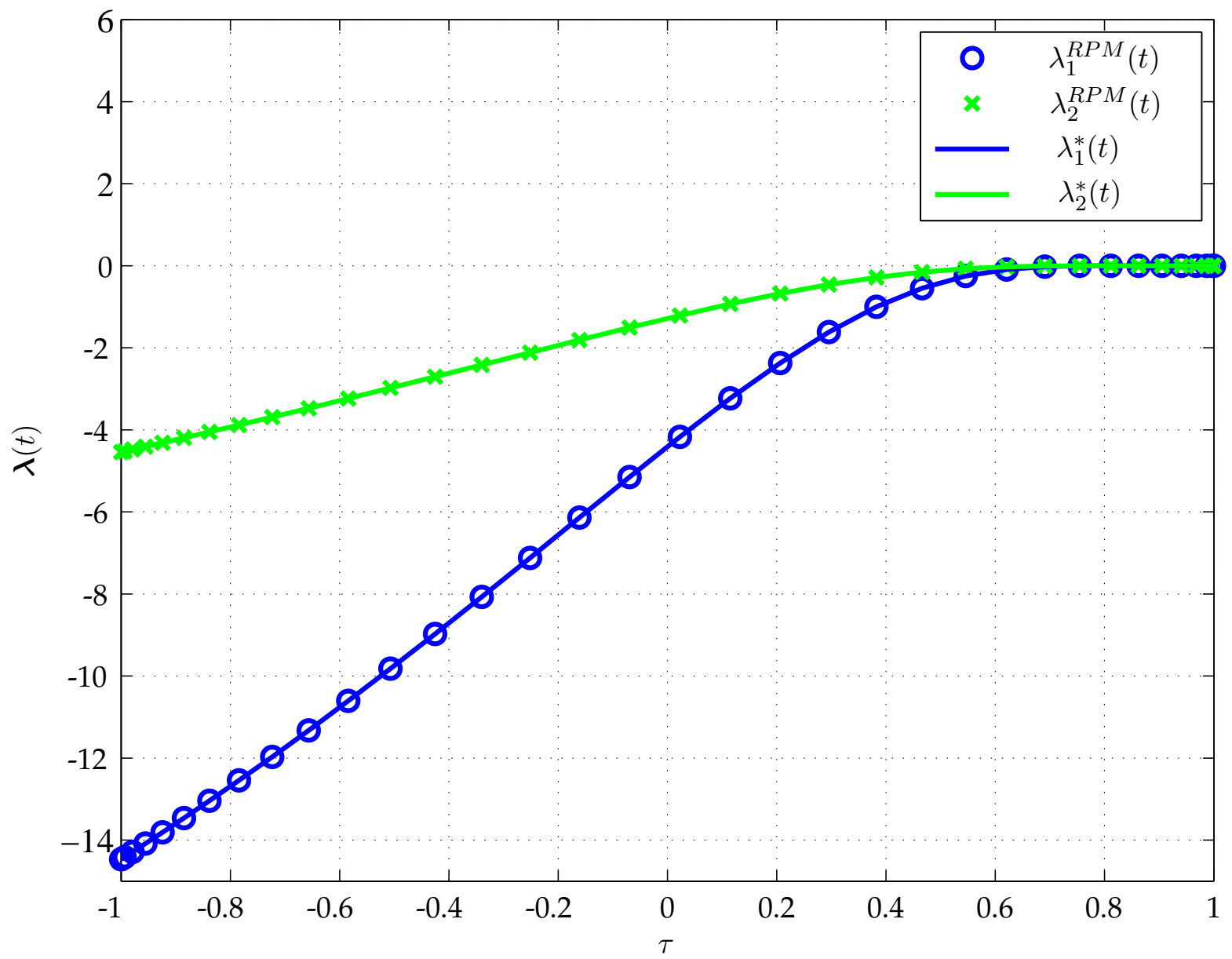

Figure 8: Infinite-Horizon Radau Pseudospectral Costate Solution for Example 2 Using $N=35$ as a Function of $\tau$ Alongside Exact Solution. 


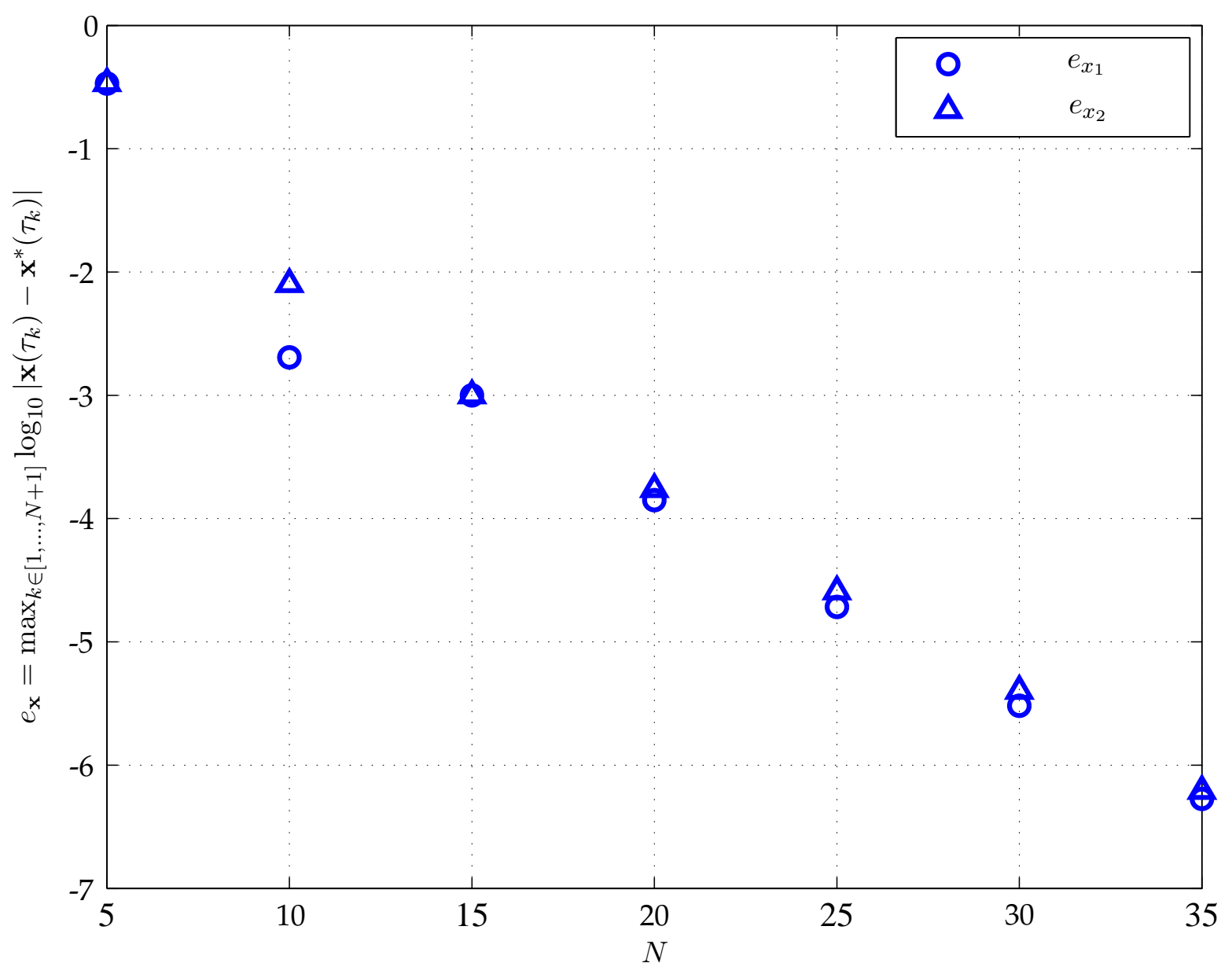

Figure 9: Error in Infinite-Horizon Radau Pseudospectral State Solution as a function of $N$. 


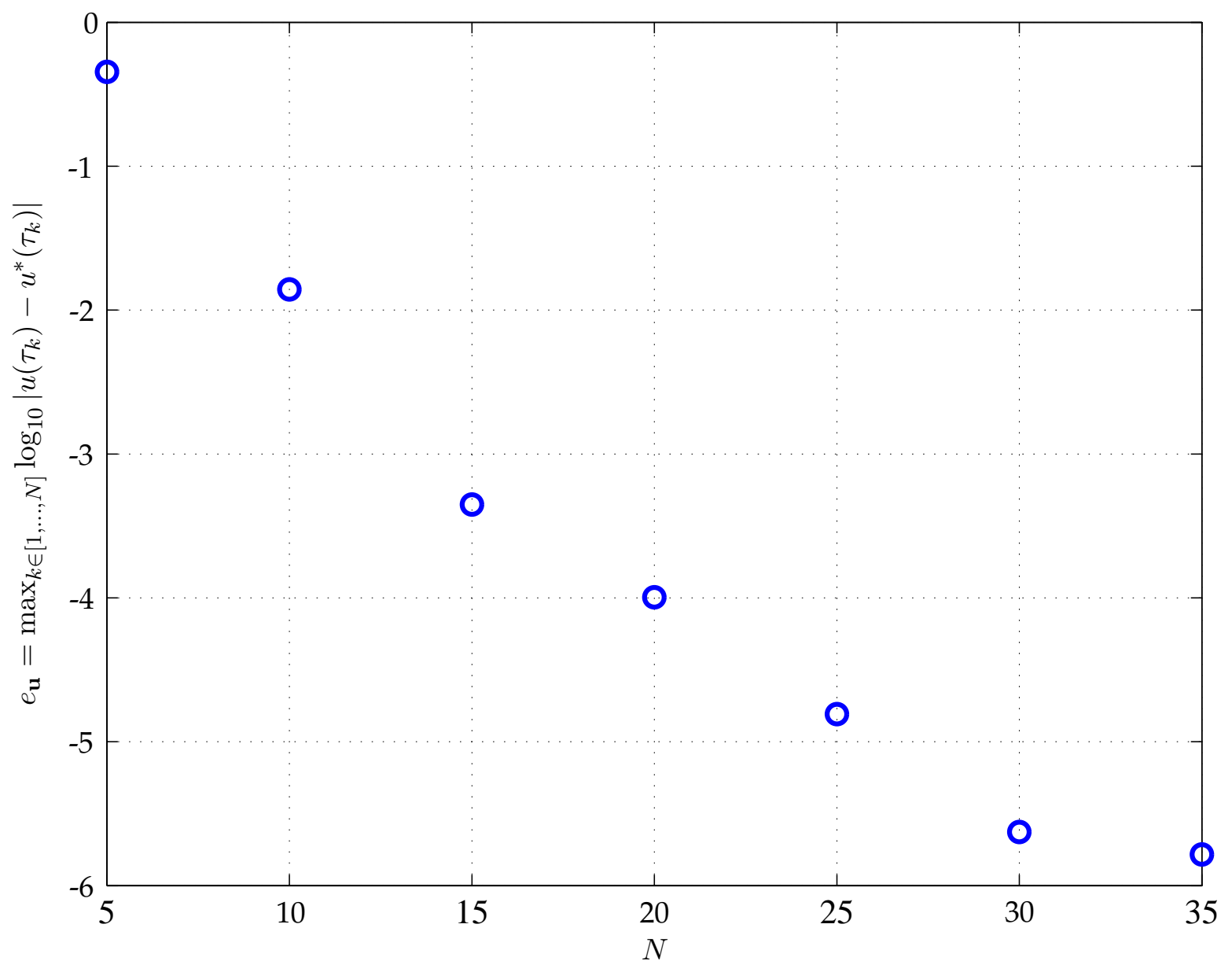

Figure 10: Error in Infinite-Horizon Radau Pseudospectral Control Solution as a function of $N$. 


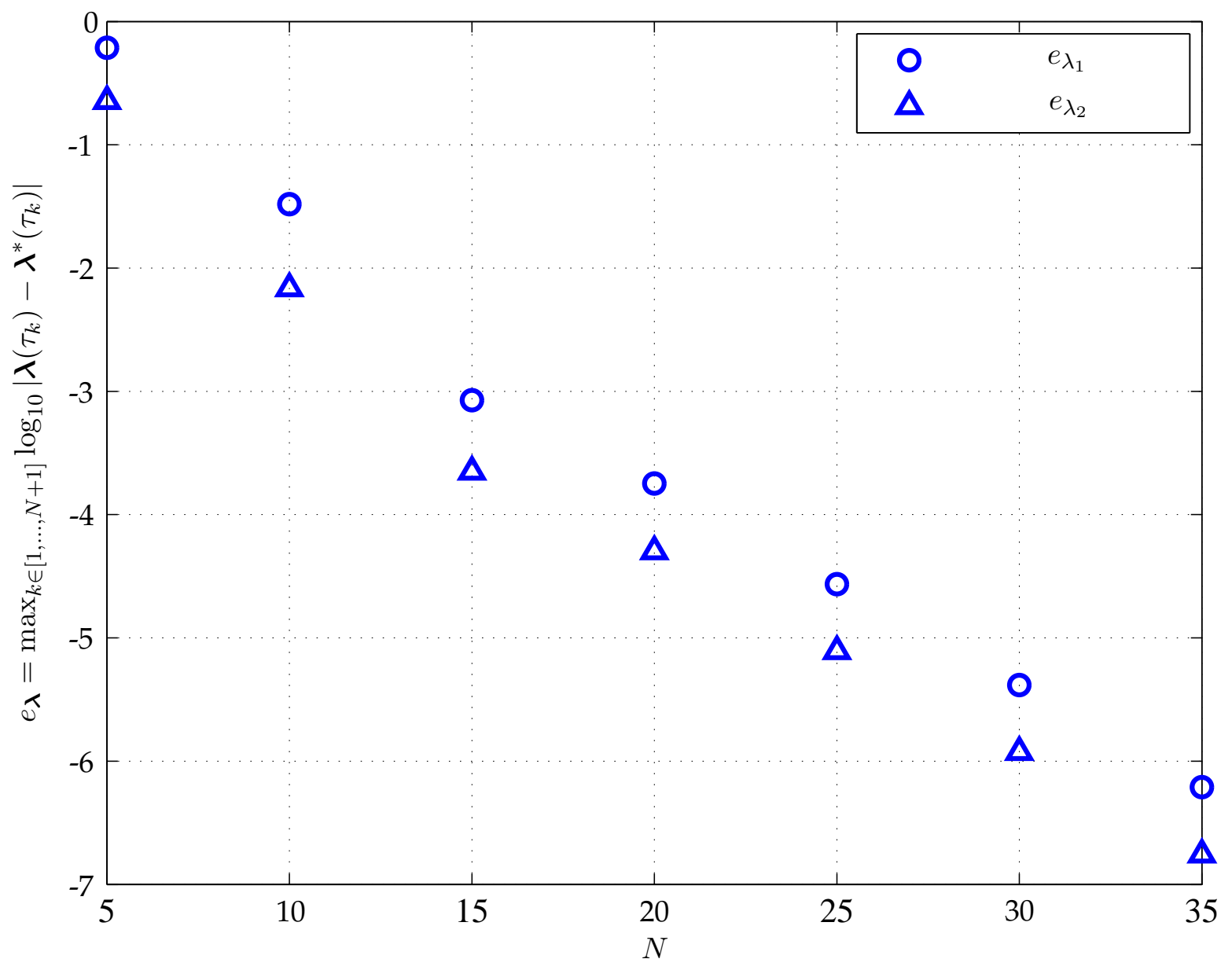

Figure 11: Error in Infinite-Horizon Radau Pseudospectral Costate Solution as a function of $N$. 
has both a larger number of variables (i.e., additional variables at the endpoints of the intervals) and a larger number of constraints (i.e., constraints required to connect the subintervals). Moreover, the method of Ref. 18 is implemented more in the form of an implicit Runge-Kutta method (due to the fact that the time interval is divided into many subintervals) whereas the method derived in this paper is implemented in the form of a pseudospectral method. It is noted that both approaches are valid, but the current approach is consistent with the manner in which pseudospectral methods have been implemented over the past several years in the aerospace control literature.

\section{B. Comparison with Global Infinite-Horizon LGR Collocation Method of Ref. 12}

While the Radau pseudospectral method derived in this paper has some similarities with the method of Ref. 12, it is important to point out that these two methods are much more different that they are alike. First, in the method presented here, the differentiation matrix is full-rank because the state is approximated using a polynomial of degree $N-1$, thereby resulting in $N-1$ independent equations for the time derivative of the state. Moreover, because the Radau differentiation matrix is full-rank, it is equivalent to the Radau integration matrix, thereby enabling the approach of this paper to be thought of as either a global implicit integration method or a pseudospectral method. Second, because all of the points (i.e., the LGR points plus the terminal point) are used to approximate the state, the method derived in this paper enables solving either finitehorizon or infinite-horizon optimal control problems. On the other hand, in the method of Ref. 12, the Radau differentiation matrix is singular.

If one wants to include boundary conditions and both ends and use the correct points at which to enforce the boundary conditions, the time derivative of the state must be a polynomial of degree $N-1$. Because Ref. 12 uses an approximation for the state that is one degree lower than is used in the method derived in this paper, Ref. 12 is limited to infinite-horizon problems (or problems that have boundary conditions at only one endpoint). Moreover, because the method of Ref. 12 does not incorporate the actual terminal point (i.e., the final point in the method of Ref. 12 is the last LGR point which is strictly less than unity), any terminal boundary condition would be applied at the incorrect point. Second, a by-product of our method using a polynomial of degree $N-1$ to approximate the state leads to the ability to construct a complete (i.e., the LGR points plus the terminal point) mapping between the indirect and direct forms. Furthermore, it was derived that the indirect and direct forms are nearly identical, the only discrepancy being the mixture of the initial transversality condition and the collocation of the dynamic constraint at the initial time as given in Eq. (21). Finally, it was shown by example that the discrepancy of Eq. (21) is small, thereby still providing a highly accurate costate approximation. Thus, while the differences between our method and the method of Ref. 12 appear to be small, the mathematical basis of our formulation is quite different from that of Ref. 12. As a result, it is important to not look just at what may appear to be subtle differences between our method and that of Ref. 12, but to see that the two forms lead to significantly different results where our method is capable of solving a wider range of problems than the method of Ref. 12.

\section{Conclusions}

A method has been presented for direct trajectory optimization and costate estimation using global collocation at Legendre-Gauss-Radau (LGR) points. A theoretical foundation for the method has been provided where the method can be constructed either as a global implicit integration method or a pseudospectral method. Using the pseudospectral (i.e., differential) form, 
it is possible to solve general finite-horizon optimal control problems and construct a complete mapping between the indirect and direct forms. In particular, the KKT multipliers of the nonlinear programming problem (NLP) can be mapped to the costates of the optimal control problem. While not exact, the costate mapping is found to be highly accurate due to the fact that the discrepancy between the indirect and direct forms is small. The method presented in this paper has been demonstrated on both a finite-horizon and infinite-horizon, thereby demonstrating the range of its utility. The results of this paper show that the Radau pseudospectral method described in this paper leads to the ability to determine accurate primal and dual solutions to general finite-horizon optimal control problems.

\section{References}

Elnagar, G., Kazemi, M., and Razzaghi, M., “The Pseudospectral Legendre Method for Discretizing Optimal Control Problems," IEEE Transactions on Automatic Control, Vol. 40, No. 10, 1995, pp. 1793-1796.

Elnagar, G. and Kazemi, M., "Pseudospectral Chebyshev Optimal Control of Constrained Nonlinear Dynamical Systems," Computational Optimization and Applications, Vol. 11, No. 2, 1998, pp. 195-217.

Fahroo, F. and Ross, I. M., "A Spectral Patching Method for Direct Trajectory Optimization," Journal of Astronautical Sciences, Vol. 48, No. 2-3, Apr.-Sept. 2000, pp. 269-286.

Fahroo, F. and Ross, I. M., "Costate Estimation by a Legendre Pseudospectral Method," Journal of Guidance, Control, and Dynamics, Vol. 24, No. 2, 2001, pp. 270-277.

Ŕoss, I. M. and Fahroo, F., Lecture Notes in Control and Information Sciences, chap. Legendre Pseudospectral Approximations of Optimal Control Problems, Springer-Verlag, 2003.

Rao, A. V., "Extension of a Pseudospectral Legendre Method for Solving Non-Sequential Multiple-Phase Optimal Control Problems," AIAA Guidance, Navigation, and Control Conference, AIAA Paper 2003-5634, Austin, Texas, August 11-14 2003.

Williams, P., “Jacobi Pseudospectral Method for Solving Optimal Control Problems," Journal of Guidance, Control, and Dynamics, Vol. 27, No. 2, 2004, pp. 293-297.

Williams, P., "Application of Pseudospectral methods for Receding Horizon Control," Journal of Guidance, Control, and Dynamics, Vol. 27, No. 2, 2004, pp. 310-314.

Pvilliams, P., "Hermite-Legendre-Gauss-Lobatto Direct Transcription Methods in Trajectory Optimization," Americal Astronautical Society, Spaceflight Mechanics Meeting, August 2005.

${ }^{1}$ Ross, I. M. and Fahroo, F., "Pseudospectral Knotting Methods for Solving Optimal Control Problems," Journal of Guidance, Control, and Dynamics, Vol. 27, No. 3, 2004, pp. 397-405.

${ }^{1}$ Fahroo, F. and Ross, I. M., “On Discrete-Time Optimality Conditions for Pseudospectral Methods," AIAA Guidance, Navigation, and Control Conference, AIAA Paper 2006-6304, Keystone, Colorado, August 2006.

1 Fahroo, F. and Ross, I. M., "Pseudospectral Methods for Infinite-Horizon Nonlinear Optimal Control Problems," Journal of Guidance, Control, and Dynamics, Vol. 31, No. 4, 2008, pp. 927-936.

${ }^{1}$ Ross, I. M. and Fahroo, F., "Advances in Pseusospectral Methods for Optimal Control," AIAA Guidance, Navigation, and Control Conference, AIAA Paper 2008-7309, Honolulu, Hawaii, August 2008.

${ }^{1}$ Ross, I. M. and Fahroo, F., "Convergence of the Costates Do Not Imply Convergence of the Controls," Journal of Guidance, Control, and Dynamics, Vol. 31, No. 4, 2008, pp. 1492-1497.

${ }^{1}$ Benson, D. A., A Gauss Pseudospectral Transcription for Optimal Control, Ph.D. thesis, MIT, 2004.

${ }^{1}$ Benson, D. A., Huntington, G. T., Thorvaldsen, T. P., and Rao, A. V., “Direct Trajectory Optimization and Costate Estimation via an Orthogonal Collocation Method," Journal of Guidance, Control, and Dynamics, Vol. 29, No. 6, NovemberDecember 2006, pp. 1435-1440.

${ }^{1}$ Huntington, G. T., Advancement and Analysis of a Gauss Pseudospectral Transcription for Optimal Control, Ph.D. thesis, Department of Aeronautics and Astronautics, Massachusetts Institute of Technology, 2007.

${ }^{1}$ Kameswaran, S. and Biegler, L. T., "Convergence Rates for Direct Transcription of Optimal Control Problems Using Collocation at Radau Points," Computational Optimization and Applications, Vol. 41, No. 1, 2008, pp. 81-126.

${ }^{1}$ Reddien, G. W., "Collocation at Gauss Points as a Discretization in Optimal Control," SIAM Journal on Control and Optimization, Vol. 17, No. 2, March 1979.

${ }^{2}$ Cuthrell, J. E. and Biegler, L. T., “On the Optimization of Differential-Algebraic Processes," AIChe Journal, Vol. 33, No. 8, August 1987, pp. 1257-1270.

28 of 29

American Institute of Aeronautics and Astronautics 
2שuthrell, J. E. and Biegler, L. T., "Simultaneous Optimization and Solution Methods for Batch Reactor Control Profiles," Computers and Chemical Engineering, Vol. 13, No. 1/2, 1989, pp. 49-62.

${ }^{2}$ Huntington, G. T., Benson, D. A., and Rao, A. V., "Optimal Configuration of Tetrahedral Spacecraft Formations," The Journal of the Astronautical Sciences, Vol. 55, No. 2, April-June 2007, pp. 141-169.

${ }^{2}$ Huntington, G. T. and Rao, A. V., “Optimal Reconfiguration of Spacecraft Formations Using the Gauss Pseudospectral Method," Journal of Guidance, Control, and Dynamics, Vol. 31, No. 3, May-June 2008, pp. 689-698.

${ }^{2}$ Kirk, D. E., Optimal Control Theory: An Introduction, Dover Publications, 2004.

${ }^{2}$ Bryson, A. E. and Ho, Y.-C., Applied Optimal Control, Hemisphere Publishing, New York, 1975.

2 Patterson, M. A., “OptimalPrime: A MATLAB Software for Solving Non-Sequential Multiple-Phase Optimal Control Problems Using Pseudospectral Methods," Dept. of Mechanical and Aerospace Engineering, University of Florida, August 2008.

2Gill, P. E., Murray, W., and Saunders, M. A., User's Guide for SNOPT Version 7: Software for Large Scale Nonlinear Programming, February 2006.

28 Won Winckel, G., collocD: Pseudospectral Differentiation on an Arbitrary Grid, The Mathworks, Inc., Central File Exchange, July 2004. 